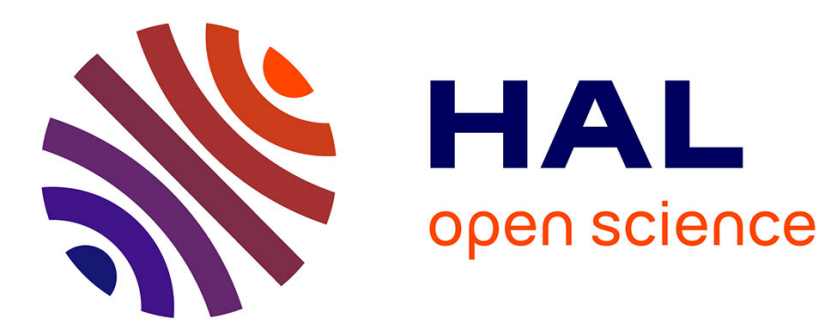

\title{
Coherence of coupling Riemann solvers for gas flows through flux-maximizing valves
}

\author{
Massimiliano D. Rosini, Andrea Corli
}

\section{To cite this version:}

Massimiliano D. Rosini, Andrea Corli. Coherence of coupling Riemann solvers for gas flows through flux-maximizing valves. 2019. hal-02099052

\section{HAL Id: hal-02099052 https://hal.science/hal-02099052}

Preprint submitted on 13 Apr 2019

HAL is a multi-disciplinary open access archive for the deposit and dissemination of scientific research documents, whether they are published or not. The documents may come from teaching and research institutions in France or abroad, or from public or private research centers.
L'archive ouverte pluridisciplinaire HAL, est destinée au dépôt et à la diffusion de documents scientifiques de niveau recherche, publiés ou non, émanant des établissements d'enseignement et de recherche français ou étrangers, des laboratoires publics ou privés. 


\title{
Coherence of coupling Riemann solvers for gas flows through flux-maximizing valves
}

\author{
Andrea Corli, Massimiliano D. Rosini \\ Department of Mathematics and Computer Science, University of Ferrara \\ I-44121 Italy
}

April 13, 2019

\begin{abstract}
In this paper we propose a model, based on the strictly hyperbolic system of isothermal Euler equations, for the gas flow in a straight pipe with a valve. We are then faced with an initial value problem with coupling conditions at the valve position. The valves under consideration are requested to maximize the flux; moreover, the flow is imposed to occur within prescribed bounds of pressure and flow. The issue is the mathematical characterization of the coherence of the corresponding coupling Riemann solvers; this property is related to the phenomenon of chattering, the rapid switch on and off of the valve. Within this framework we describe three kinds of valves, which differ for their action; two of them lead to a coherent solver, the third one does not. Proofs involve geometric and analytic properties of the Lax curves.
\end{abstract}

Keywords: systems of conservation laws, gas flow, valve, Riemann problem, coupling conditions.

2010 AMS subject classification: 35L65, 35L67, 76B75.

\section{Introduction}

In this paper we investigate some qualitative properties of the gas flow through a valve in a straight pipe. The gas is assumed to fill the whole pipe and its velocity to be constant on every cross-section; moreover, we neglect the friction exerted by the walls of the pipe. Away from the valve, the flow can be modeled by two conservation laws expressing the conservation of mass and momentum:

$$
\left\{\begin{array}{l}
\partial_{t} \rho+\partial_{x}(\rho v)=0, \\
\partial_{t}(\rho v)+\partial_{x}\left(\rho v^{2}+p(\rho)\right)=0 .
\end{array}\right.
$$

Here $t \geqslant 0$ represents the time, $x \in \mathbb{R}$ the space position along the pipe, $\rho>0$ the mass density of the gas and $v \in \mathbb{R}$ its velocity. By discarding thermal effects we assume that the pressure $p$ is given by

$$
p(\rho) \doteq a^{2} \rho,
$$

for $a>0$. We refer to $[2,3,6,15]$ for the use of equations $(1.1),(1.2)$ in analogous problems.

We assume that the valve is located at $x=0$. The fluid motion through valves is an important issue in engineering, see for instance [18] and references there; more mathematically-oriented information is contained in $[14,22,23]$. However, a satisfactory mathematical modeling based on system (1.1) is still missing; our recent papers $[7,8]$ aimed at that direction. There are indeed several ways of modeling compressible flows through valves and the most common rely on dynamical systems, see for instance [21] and references there. This modeling, however, is more concerned with the mechanical behavior of the valves under consideration; here, on the contrary, we model the valve as a "black box" and the focus is on the understanding its effects.

Before giving a brief account of $[7,8]$, we recall what coherence means for a Riemann solver. Consider a self-similar solution $u(t, x) \doteq U(x / t)$ of a Riemann problem for (1.1) and consider its traces $U\left(\xi_{o}^{-}\right)$and $U\left(\xi_{o}^{+}\right)$at any $\xi_{o} \in \mathbb{R}$. The solver is coherent if, roughly speaking, the solution to the Riemann problem with data $U\left(\xi_{0}^{-}\right)$and $U\left(\xi_{o}^{+}\right)$locally coincides with $u$; a more precise definition is given below. Any Lax 
Riemann-solver is coherent. We point out that coherence is a necessary condition for the construction of global solutions with bounded-variation initial data.

In [7] we provided a general framework for the modeling and study of valves. The valve is modeled by suitable conditions at $x=0$ and influences the flow at both $x<0$ and $x>0$. This leads to the definition of coupling Riemann solvers, which match the flows on the two sides of the valve with the action of the valve. The previous notion of coherence is easily extended to these solvers. As an application, we modeled a two-way valve that was open or closed according to a threshold of the pressure gradient. Such valves are known as pressure regulators. We proposed a coupling Riemann solver and noticed that coherence could be lost because of the presence of the valve. As a consequence, we studied the coherence, consistence, continuity with respect to the initial data and invariant domains of the solver. Analogous results were specialized in [8] to the case of one-way valves, where the gas only flows in one direction. As an example, we studied a valve that aims at keeping a fixed outgoing flow $q_{*}>0$; when this is not possible, then the valve shuts. Such valves are known as pressure independent characterized control valves.

We also constructed in [7] invariant domains where coherence is satisfied; analogous domains, but only consisting of subsonic states, were constructed in [8]. This last result was motivated by the fact that gas flows through pipes are usually subsonic. We stress that, in general, subsonic flows do not guarantee coherence [7]. Moreover, supersonic states may arise even if the initial datum only involves subsonic states and no valve is present; this follows from the fact that any non-trivial invariant domain for the Lax Riemann-solver contains supersonic states. As we showed in [8], the lack of coherence corresponds to the phenomenon of chattering, the rapid and repeated opening and closing of the valve. We refer to [16] for a study of chattering in the framework of the modeling of the valve by dynamical systems. The aim of $[7,8]$ and of the current paper is to lay the foundations for the mathematical study of valves in order to investigate, in future papers, the flow control by the valve parameters to avoid the chattering. We emphasize that our approach is sufficiently general to include the case when a compressor replaces a valve; we refer to [15] for such a case.

As we mentioned above, in $[7,8]$ we investigated in particular the invariant domains of several coupling Riemann solvers corresponding to gas flows through valves. In this paper we reverse the point of view: we search for general conditions on a valve ensuring that the states in $x<0$ and $x>0$ satisfy suitable bounds of pressure and flow for initial states in those ranges. Such ranges are modeled by invariant domains. This mere requirement does not clearly single out a solution to the Riemann problem but leaves room for specific valve models that fit for this framework. A challenging problem of gas flows in networks is the optimization of the flux, in order to improve efficiency and increase throughput. Having this issue in mind, here we consider a general model of valve that allows for the highest possible volume flow.

The paper is organized as follows. In the next section we briefly give some preliminary results; they partially overlap with analogous material in $[7,8]$, but are necessary to understand the following. In Section 3 we introduce our general modeling of flows through valves. In Section 4 we impose a condition of maximization of the flow through the valve and propose three models of valves, which differ for the sets where the valves are active; the main issue is the coherence of the corresponding coupling Riemann solvers. In a first case coherence holds while in a second it does not. The third example matches these two extreme cases: by a suitable limiting process we explicitly construct a set, which is minimal for the relation of inclusion, where the valve is active and the corresponding Riemann solver is coherent. Some technical proofs are deferred to Section 5.

\section{Preliminary results and notation}

In this section we provide some background results and give several definitions. System (1.1) with the pressure law (1.2) can be written in the conservative $(\rho, q)$-variables, with $q \doteq \rho v$, as

$$
\left\{\begin{array}{l}
\partial_{t} \rho+\partial_{x} q=0, \\
\partial_{t} q+\partial_{x}\left(\frac{q^{2}}{\rho}+a^{2} \rho\right)=0 .
\end{array}\right.
$$


We denote $u \doteq(\rho, q)$; then $u$ takes values in $\Omega \doteq\left\{(\rho, q) \in \mathbb{R}^{2}: \rho>0\right\}$. We recall that the Riemann problem for $(2.1)$ is the initial-value problem with initial condition

$$
u(0, x)=\left\{\begin{array}{ll}
u_{\ell} & \text { if } x<0, \\
u_{r} & \text { if } x \geqslant 0,
\end{array} \quad u_{\ell}, u_{r} \in \Omega .\right.
$$

We denote $\mathbb{R}_{-} \doteq(-\infty, 0]$ and $\mathbb{R}_{+} \doteq[0, \infty)$.

Definition 2.1. A function $u \in \mathbf{C}^{\mathbf{0}}\left(\mathbb{R}_{+} ; \mathbf{L}^{\infty}(\mathbb{R} ; \Omega)\right)$ is a weak solution of Riemann problem (2.1), (2.2) in $\mathbb{R}_{+} \times \mathbb{R}$ if for any $\varphi \in \mathbf{C}_{\mathbf{c}}^{\infty}\left(\mathbb{R}_{+} \times \mathbb{R} ; \mathbb{R}\right)$ we have

$$
\begin{array}{r}
\iint_{\mathbb{R}_{+} \times \mathbb{R}}\left(\rho \partial_{t} \varphi+q \partial_{x} \varphi\right) \mathrm{d} x \mathrm{~d} t+\rho_{\ell} \int_{\mathbb{R}_{-}} \varphi(0, x) \mathrm{d} x+\rho_{r} \int_{\mathbb{R}_{+}} \varphi(0, x) \mathrm{d} x=0, \\
\iint_{\mathbb{R}_{+} \times \mathbb{R}^{2}}\left(q \partial_{t} \varphi+\left(\frac{q^{2}}{\rho^{2}}+a^{2}\right) \rho \partial_{x} \varphi\right) \mathrm{d} x \mathrm{~d} t+q_{\ell} \int_{\mathbb{R}_{-}} \varphi(0, x) \mathrm{d} x+q_{r} \int_{\mathbb{R}_{+}} \varphi(0, x) \mathrm{d} x=0 .
\end{array}
$$

We denote by $\mathbf{B V}(\mathbb{R} ; \Omega)$ the space of $\Omega$-valued functions with bounded variation. We can assume that any function in $\mathbf{B V}(\mathbb{R} ; \Omega)$ is right continuous by possibly changing its values at countably many points.

Definition 2.2. Let $\mathrm{D} \subseteq \Omega \times \Omega$ and $\mathcal{R S}: \mathrm{D} \rightarrow \mathbf{B V}(\mathbb{R} ; \Omega)$.

- We say that $\mathcal{R S}$ is a Riemann solver for (2.1) if for any $\left(u_{\ell}, u_{r}\right) \in \mathrm{D}$ the self-similar function $u(t, x) \doteq$ $\mathcal{R S}\left[u_{\ell}, u_{r}\right](x / t)$ is a weak solution to $(2.1),(2.2)$ in $\mathbb{R}_{+} \times \mathbb{R}$.

- A Riemann solver $\mathcal{R S}$ is coherent at $\left(u_{\ell}, u_{r}\right) \in \mathrm{D}$ if $U \doteq \mathcal{R} \mathcal{S}\left[u_{\ell}, u_{r}\right]$ satisfies for any $\xi_{o} \in \mathbb{R}$

$$
\left(U\left(\xi_{o}^{-}\right), U\left(\xi_{o}^{+}\right)\right) \in \mathrm{D} \quad \text { and } \quad \mathcal{R S}\left[U\left(\xi_{o}^{-}\right), U\left(\xi_{o}^{+}\right)\right](\xi)= \begin{cases}U\left(\xi_{o}^{-}\right) & \text {if } \xi<\xi_{o} \\ U\left(\xi_{o}^{+}\right) & \text {if } \xi \geq \xi_{o}\end{cases}
$$

We say that $\mathcal{R S}$ is coherent if it is coherent in the whole of $\mathrm{D}$.

- A subset $\mathcal{I}$ of $\Omega$ is an invariant domain for $\mathcal{R S}$ if $\mathcal{I} \times \mathcal{I} \subseteq \mathrm{D}$ and $\mathcal{R S}[\mathcal{I}, \mathcal{I}](\mathbb{R}) \subseteq \mathcal{I}$.

According to the previous definition, a Riemann solver $\mathcal{R S}$ is coherent at an initial datum $\left(u_{\ell}, u_{r}\right) \in \mathrm{D}$ if the ordered pair $\left(\mathcal{R S}\left[u_{\ell}, u_{r}\right]\left(\xi_{o}^{-}\right), \mathcal{R} \mathcal{S}\left[u_{\ell}, u_{r}\right]\left(\xi_{o}^{+}\right)\right)$of the traces of the corresponding solution belongs to $\mathrm{D}$ and, in a sense, is a fixed point for $\mathcal{R} \mathcal{S}$.

The coherence of a Riemann solver is a desirable property for a numerical scheme. In fact, a numerical time-stepping scheme, based on a Riemann solver that fails to be consistent, may not produce the expected solution of a Riemann problem $[8,10]$. We notice that the lack of coherence has a physical counterpart, which is typical when dealing with real valves: it can induce chattering [8].

Coherence may fail in presence of a valve, see [7, 8] and Subsection 4.2 below. However, the Lax Riemann-solver $\mathcal{R S}_{\mathrm{p}}: \Omega \times \Omega \rightarrow \mathbf{B V}(\mathbb{R} ; \Omega)$ of (2.1), see [20], is coherent [7, Proposition 2.5]. We denote for any $x \in \mathbb{R}$ and $t \in \mathbb{R}_{+}$

$$
u_{\mathrm{p}}(t, x) \doteq \mathcal{R} \mathcal{S}_{\mathrm{p}}\left[u_{\ell}, u_{r}\right](x / t), \quad u_{\mathrm{p}}^{ \pm} \doteq u_{\mathrm{p}}\left(t, 0^{ \pm}\right)
$$

System (2.1) is strictly hyperbolic in $\Omega$ and both eigenvalues $\lambda_{1}(u) \doteq v-a, \lambda_{2}(u) \doteq v+a$ are genuinely nonlinear [20]. Any smooth discontinuity curve $x=\gamma(t)$ of a weak solution $u$ of (2.1) satisfies the RankineHugoniot conditions

$$
\begin{aligned}
& \left(\rho^{+}-\rho^{-}\right) \dot{\gamma}=q^{+}-q^{-}, \\
& \left(q^{+}-q^{-}\right) \dot{\gamma}=\left(\frac{\left(q^{+}\right)^{2}}{\rho^{+}}+a^{2} \rho^{+}\right)-\left(\frac{\left(q^{-}\right)^{2}}{\rho^{-}}+a^{2} \rho^{-}\right),
\end{aligned}
$$

where $u^{ \pm}(t) \doteq u\left(t, \gamma(t)^{ \pm}\right)$are the traces of $u$, see $[5,9]$. 
For any fixed $u_{o} \in \Omega$ we define $\mathcal{S}_{i}^{u_{o}}, \mathcal{R}_{i}^{u_{o}}:(0, \infty) \rightarrow \mathbb{R}, i \in\{1,2\}$, as

$$
\mathcal{S}_{i}^{u_{o}}(\rho) \doteq \rho\left[\frac{q_{o}}{\rho_{o}}+(-1)^{i} a\left(\sqrt{\frac{\rho}{\rho_{o}}}-\sqrt{\frac{\rho_{o}}{\rho}}\right)\right], \quad \mathcal{R}_{i}^{u_{o}}(\rho) \doteq \rho\left[\frac{q_{o}}{\rho_{o}}+(-1)^{i} a \log \left(\frac{\rho}{\rho_{o}}\right)\right] .
$$

Then we define $\mathcal{F} \mathcal{L}_{i}^{u_{o}}, \mathcal{B} \mathcal{L}_{i}^{u_{o}}:(0, \infty) \rightarrow \mathbb{R}, i \in\{1,2\}$, by

$$
\begin{aligned}
\mathcal{F} \mathcal{L}_{1}^{u_{o}}(\rho) \doteq \begin{cases}\mathcal{R}_{1}^{u_{o}}(\rho) & \text { if } \rho \in\left(0, \rho_{o}\right], \\
\mathcal{S}_{1}^{u_{o}}(\rho) & \text { if } \rho \in\left(\rho_{o}, \infty\right),\end{cases} & \mathcal{F} \mathcal{L}_{2}^{u_{o}}(\rho) \doteq \begin{cases}\mathcal{S}_{2}^{u_{o}}(\rho) & \text { if } \rho \in\left(0, \rho_{o}\right), \\
\mathcal{R}_{2}^{u_{o}}(\rho) & \text { if } \rho \in\left[\rho_{o}, \infty\right),\end{cases} \\
\mathcal{B \mathcal { L }}_{1}^{u_{o}}(\rho) \doteq \begin{cases}\mathcal{S}_{1}^{u_{o}}(\rho) & \text { if } \rho \in\left(0, \rho_{o}\right), \\
\mathcal{R}_{1}^{u_{o}}(\rho) & \text { if } \rho \in\left[\rho_{o}, \infty\right),\end{cases} & \mathcal{B L}_{2}^{u_{o}}(\rho) \doteq \begin{cases}\mathcal{R}_{2}^{u_{o}}(\rho) & \text { if } \rho \in\left(0, \rho_{o}\right], \\
\mathcal{S}_{2}^{u_{o}}(\rho) & \text { if } \rho \in\left(\rho_{o}, \infty\right)\end{cases}
\end{aligned}
$$

The graphs of the functions $\mathcal{F} \mathcal{L}_{i}^{u_{o}}$ and $\mathcal{B L}_{i}^{u_{o}}$ are the forward $\mathrm{FL}_{i}^{u_{o}}$ and backward $\mathrm{BL}_{i}^{u_{o}}$ Lax curves of the $i$-th family through $u_{o}$, see Figure 1 . We stress that all pictures in this paper are constructed with exact Lax curves. Analogously, the shock $\mathrm{S}_{i}^{u_{o}}$ and rarefaction $\mathrm{R}_{i}^{u_{o}}$ curves through $u_{o}$ are the graphs of the functions $\mathcal{S}_{i}^{u_{o}}$ and $\mathcal{R}_{i}^{u_{o}}$. The shock speeds are $s_{i}^{u_{o}}(\rho) \doteq v_{o}+(-1)^{i} a \sqrt{\rho / \rho_{o}}, i \in\{1,2\}$.
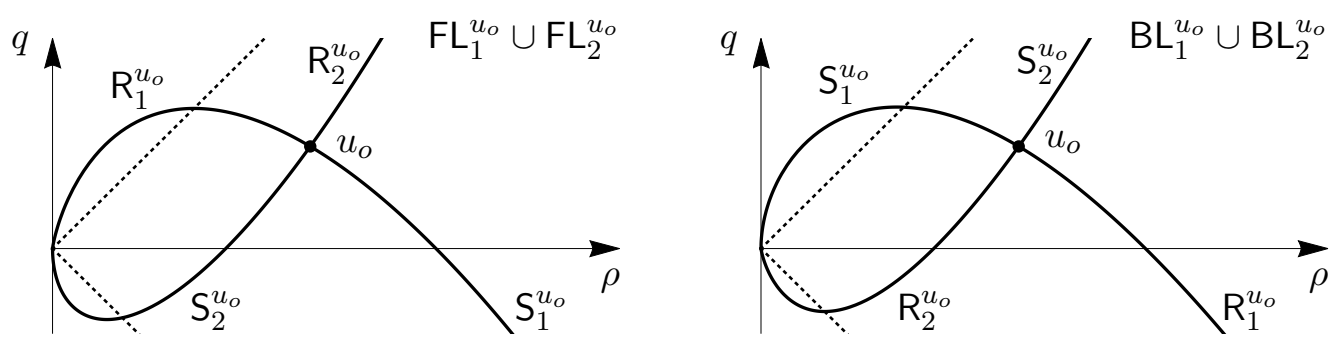

Figure 1: Forward and backward Lax curves. Here and in the following the dotted straight lines are the sonic lines $q= \pm a \rho$.

We now provide the basic properties of the sets $S_{i}^{u_{o}}, \mathrm{R}_{i}^{u_{o}}$, see [8, Proposition 2.3].

Proposition 2.3. Let $u_{o}, u^{o} \in \Omega$ be two distinct states and $i \in\{1,2\}$. Then we have:

(L1) $\mathrm{R}_{i}^{u_{o}} \cap \mathrm{R}_{i}^{u^{o}} \neq \emptyset$ if and only if $\mathrm{R}_{i}^{u_{o}}=\mathrm{R}_{i}^{u^{o}}$;

(L2) $\mathrm{S}_{i}^{u_{o}} \cap \mathrm{S}_{i}^{u^{o}}$ has at most two elements, hence if $u^{o} \in \mathrm{S}_{i}^{u_{o}}$ then $\mathrm{S}_{i}^{u^{o}} \cap \mathrm{S}_{i}^{u_{o}}=\left\{u^{o}, u_{o}\right\}$;

(L3) $\mathrm{R}_{1}^{u_{o}}, \mathrm{~S}_{1}^{u_{o}}, \mathrm{FL}_{1}^{u_{o}}$ and $\mathrm{BL}_{1}^{u_{o}}$ are strictly concave, while $\mathrm{R}_{2}^{u_{o}}, \mathrm{~S}_{2}^{u_{o}}, \mathrm{FL}_{2}^{u_{o}}$ and $\mathrm{BL}_{2}^{u_{o}}$ are strictly convex;

(L4) we have, see Figure 2,

$$
\left\{\begin{array}{l}
\mathcal{S}_{2}^{u_{o}}(\rho)=\mathcal{F} \mathcal{L}_{2}^{u_{o}}(\rho)<\mathcal{R}_{2}^{u_{o}}(\rho)=\mathcal{B L}_{2}^{u_{o}}(\rho)<\mathcal{R}_{1}^{u_{o}}(\rho)=\mathcal{F} \mathcal{L}_{1}^{u_{o}}(\rho)<\mathcal{S}_{1}^{u_{o}}(\rho)=\mathcal{B L}_{1}^{u_{o}}(\rho) \quad \text { if } \rho<\rho_{o}, \\
\mathcal{S}_{1}^{u_{o}}(\rho)=\mathcal{F} \mathcal{L}_{1}^{u_{o}}(\rho)<\mathcal{R}_{1}^{u_{o}}(\rho)=\mathcal{B L}_{1}^{u_{o}}(\rho)<\mathcal{R}_{2}^{u_{o}}(\rho)=\mathcal{F} \mathcal{L}_{2}^{u_{o}}(\rho)<\mathcal{S}_{2}^{u_{o}}(\rho)=\mathcal{B L}_{2}^{u_{o}}(\rho) \quad \text { if } \rho>\rho_{o}
\end{array}\right.
$$

(L5) $\frac{\mathrm{d} \mathcal{S}_{i}^{u_{o}}}{\mathrm{~d} \rho}\left(0^{+}\right)=(-1)^{i+1} \infty$ and $\frac{\mathrm{d} \mathcal{R}_{i}^{u_{o}}}{\mathrm{~d} \rho}\left(0^{+}\right)=(-1)^{i+1} \infty$;

(L6) $\mathcal{F} \mathcal{L}_{i}^{u_{o}}$ and $\mathcal{B} \mathcal{L}_{i}^{u_{o}}$ are $\mathbf{C}^{2}$ functions.

A state $(\rho, q)$ is called subsonic if $|v|<a$ and supersonic if $|v|>a$. The lines $q= \pm a \rho$ are called sonic lines. By referring to Figure 3, for $u_{\ell}, u_{r} \in \Omega$ we define:

- $\bar{u}\left(u_{\ell}\right)$ is the element of $\mathrm{FL}_{1}^{u_{\ell}}$ with the maximum $q$-coordinate;

- $\underline{u}\left(u_{r}\right)$ is the element of $\mathrm{BL}_{2}^{u_{r}}$ with the minimum $q$-coordinate;

- $\tilde{u}\left(u_{\ell}, u_{r}\right)$ is the (unique) element of $\mathrm{FL}_{1}^{u_{\ell}} \cap \mathrm{BL}_{2}^{u_{r}}$;

- $\hat{u}\left(q_{o}, u_{\ell}\right)$, for any $q_{o} \leqslant \bar{q}\left(u_{\ell}\right)$, is the intersection of $\mathrm{FL}_{1}^{u_{\ell}}$ and $q=q_{o}$ with the largest $\rho$-coordinate;

- $\check{u}\left(q_{o}, u_{r}\right)$, for any $q_{o} \geqslant \underline{q}\left(u_{r}\right)$, is the intersection of $\mathrm{BL}_{2}^{u_{r}}$ and $q=q_{o}$ with the largest $\rho$-coordinate.

We now show some properties of the above quantities; we denote $\left(\bar{\rho}\left(u_{\ell}\right), \bar{q}\left(u_{\ell}\right)\right) \doteq \bar{u}\left(u_{\ell}\right)$ and so on. First, notice that for any $u_{\ell}, u_{r} \in \Omega$ we have $\underline{q}\left(u_{r}\right)<0<\bar{q}\left(u_{\ell}\right)$. 

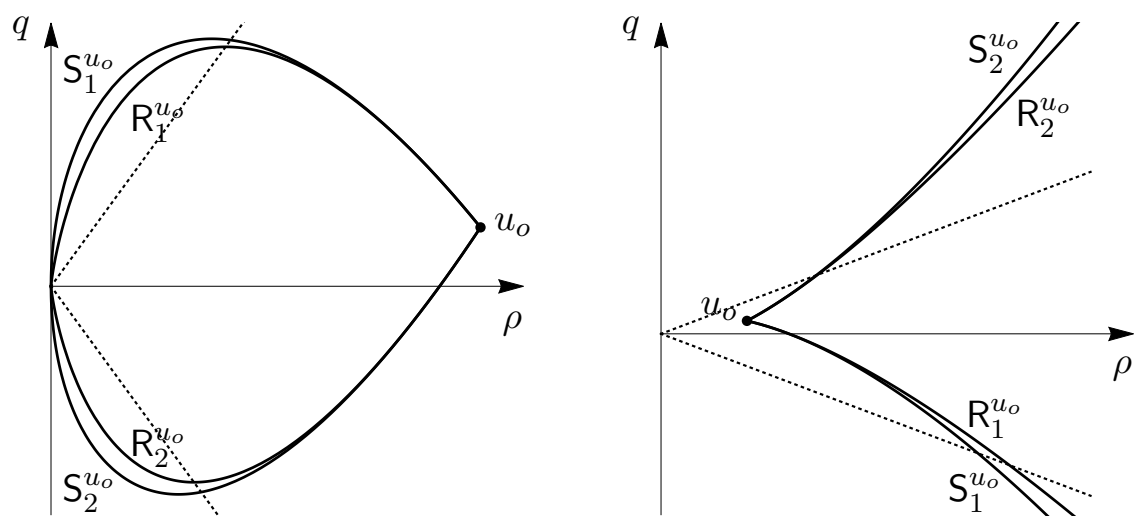

Figure 2: The curves $\mathrm{R}_{i}^{u_{o}}$ and $\mathrm{S}_{i}^{u_{o}}, i=1,2$.
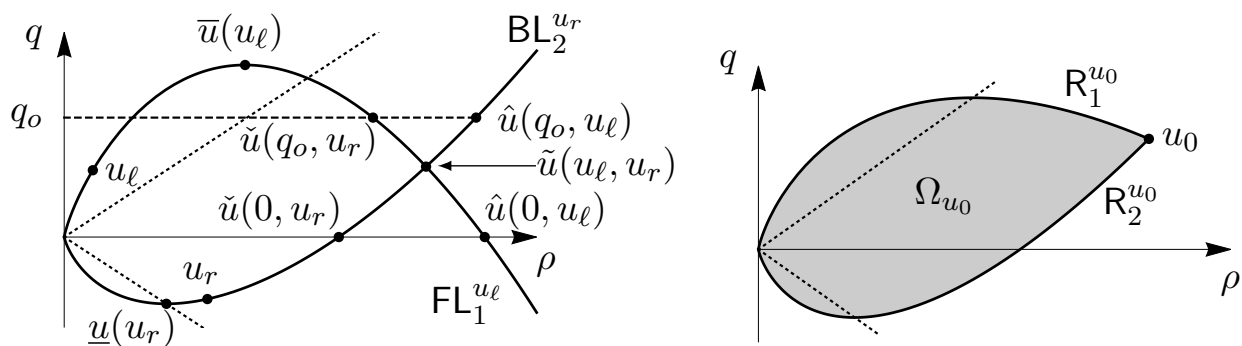

Figure 3: Left: notations. Right: a typical invariant domain $\Omega_{u_{0}}$ for $\mathcal{R} \mathcal{S}_{\mathrm{p}}$.

Lemma 2.4. We denote $v_{\ell} \doteq q_{\ell} / \rho_{\ell}$ and $v_{r} \doteq q_{r} / \rho_{r}$. Then we have:

$$
\begin{aligned}
& \rho_{\ell}>\bar{\rho}\left(u_{\ell}\right) \quad \Longleftrightarrow \quad v_{\ell}<a \quad \Longleftrightarrow \quad v_{\ell}<\bar{v}\left(u_{\ell}\right) \quad \Longrightarrow \bar{v}\left(u_{\ell}\right)=a, \\
& \rho_{\ell}<\bar{\rho}\left(u_{\ell}\right) \quad \Longleftrightarrow v_{\ell}>a \quad \Longleftrightarrow v_{\ell}>\bar{v}\left(u_{\ell}\right) \quad \Longleftrightarrow \bar{v}\left(u_{\ell}\right)>a \text {. }
\end{aligned}
$$

Analogously we have

$$
\begin{gathered}
\rho_{r}>\underline{\rho}\left(u_{r}\right) \quad \Longleftrightarrow \quad v_{r}>-a \quad \Longleftrightarrow \quad v_{r}>\underline{v}\left(u_{r}\right) \quad \Longleftrightarrow \quad \underline{v}\left(u_{r}\right)=-a, \\
\rho_{r}<\underline{\rho}\left(u_{r}\right) \quad \Longleftrightarrow \quad v_{r}<-a \quad \Longleftrightarrow \quad v_{r}<\underline{v}\left(u_{r}\right) \quad \Longleftrightarrow \quad \underline{v}\left(u_{r}\right)>-a .
\end{gathered}
$$

Expressions (2.6) and (2.7) hold true by replacing strict inequalities with equalities. At last, if $v_{\ell} \leqslant a$ then we have $\bar{v}\left(u_{\ell}\right)=a$ and $\bar{q}\left(u_{\ell}\right)=a \bar{\rho}\left(u_{\ell}\right)$, while if $v_{r} \geqslant-a$ then $\underline{v}\left(u_{r}\right)=-a$, and $\underline{q}\left(u_{r}\right)=-a \underline{\rho}\left(u_{r}\right)$.

We recall, see Figure 3 on the right, that invariant domains for $\mathcal{R} \mathcal{S}_{\mathrm{p}}$ have the form [17]

$$
\Omega_{u_{0}}=\left\{u \in \Omega: \mathcal{R}_{2}^{u_{0}}(\rho) \leqslant q \leqslant \mathcal{R}_{1}^{u_{0}}(\rho)\right\}, \quad u_{0} \in \Omega .
$$

\section{The mathematical modeling of the flow through a valve}

In this section we recall the modeling of a gas flow through a valve located at $x=0 ;$ see $[7,8]$ for more details. Differently from those papers, where the flow was either controlled by the difference of the pressure or according to a fixed value of the flow, here we consider valves that aim to keep the state variables in suitable ranges, which can be thought as characterizing the operating range of the valve. From a mathematical point of view, we identify the sets defined by these ranges as invariant domains.

More precisely, fix $u_{ \pm}^{*} \in \Omega$ and denote by $\Omega_{ \pm} \doteq \Omega_{u_{ \pm}^{*}}$ two invariant domains as in (2.8). We require that $u$ satisfies

$$
u(t, \pm x) \in \Omega_{ \pm} \quad \text { and } \quad u\left(t, 0^{ \pm}\right) \in \Omega_{ \pm} \quad \text { if } x>0 \text { and } t \geqslant 0 .
$$

Condition (3.1) ensures that the values of pressure and flow on the left and on the right of the valve are in the ranges [23]

$$
p(t, \pm x) \in\left[0, p_{ \pm}^{*}\right] \quad \text { and } \quad q(t, \pm x) \in\left[\underline{q}\left(u_{ \pm}^{*}\right), \bar{q}\left(u_{ \pm}^{*}\right)\right] \quad \text { if } x>0 \text { and } t \geqslant 0
$$


where $p_{ \pm}^{*} \doteq p\left(\rho_{ \pm}^{*}\right)$. The motivation for condition (3.1), and then (3.2), lies in the fact that high values of pressure and flow should be avoided for safety or management reasons. Notice that both conditions prescribe possibly different ranges for the two segments $x<0$ and $x>0$ of the pipe, in order to model different characteristics of the parts of the pipes.

From a physical point of view, the domain

$$
\mathrm{D}_{\mathrm{v}} \doteq \Omega_{-} \times \Omega_{+}
$$

represents the characteristic range of the two sections of the pipe. Condition (3.1) can be achieved in several ways, according to the valve model. The valve can be either inactive (bypass) or active. In the former case, system (2.1) fully describes the flow in the whole of $\mathbb{R}$ : no additional condition is imposed and the flow takes place exactly as the valve is missing. In particular, the valve is understood as "fully open". In the latter case, the valve acts as an exterior force on the flow and then the conservation of momentum may be lost; such an action is modeled by considering two coupled initial-boundary value problems in $\mathbb{R}_{+} \times \mathbb{R}_{-}$ and $\mathbb{R}_{+} \times \mathbb{R}_{+}$. On the contrary, conservation of mass still occurs. As a consequence, along $x=0$ only the first Rankine-Hugoniot condition (2.3) is imposed. The following definitions provide a general framework to this modeling.

Definition 3.1. A function $u \in \mathbf{C}^{\mathbf{0}}\left(\mathbb{R}_{+} ; \mathbf{B V}(\mathbb{R} ; \Omega)\right)$ is a coupling solution of Riemann problem (2.1), (2.2) if $u$ satisfies (3.1) and its restrictions to $x<0$ and $x>0$ are weak self-similar solutions (in the sense of $[1,4])$ to the initial-boundary value problem for (2.1) with initial data

$$
u(0, x)=u_{\ell} \quad \text { if } x<0, \quad u(0, x)=u_{r} \quad \text { if } x>0,
$$

and coupling boundary conditions

$$
q\left(t, 0^{-}\right)=q\left(t, 0^{+}\right) \quad \text { for a.e. } t>0 .
$$

We stress that the restrictions of a coupling solution $u$ to $x<0$ and $x>0$ take values respectively in $\Omega_{-}$ and $\Omega_{+}$as well as its traces. This definition extends the notion of weak solution provided in Definition 2.1. In particular, it takes into account the possible presence of stationary discontinuities of $u$ at $x=0$, which satisfy the first Rankine-Hugoniot condition (2.3) with $\dot{\gamma} \equiv 0$, that is (3.3), but not necessarily the second Rankine-Hugoniot condition (2.4). Roughly speaking, these discontinuities can also be understood as undercompressive shock waves [19], because they do not necessarily satisfy the Lax conditions. At last, notice that the boundary $x=0$ can be characteristic.

Every self-similar weak solution of $(2.1),(2.2)$ taking values in the invariant domain $\Omega_{-} \cap \Omega_{+}$is a coupling solution, but the converse is not necessarily true. This is in the same spirit of the solutions considered in $[11,12,13]$.

For (2.1) we always use the Lax Riemann-solver except at $x=0$; at $x=0$ we model the flow through the valve by a coupling Riemann solver. The extension of Definition 2.2 to this framework is the following.

Definition 3.2. Let $\mathcal{R S}: \mathrm{D}_{\mathrm{v}} \rightarrow \mathrm{BV}(\mathbb{R} ; \Omega)$.

- We say that $\mathcal{R S}$ is a coupling Riemann solver for $(2.1)$ if for any $\left(u_{\ell}, u_{r}\right) \in \mathrm{D}_{\mathrm{v}}$ the function $u(t, x) \doteq$ $\mathcal{R S}\left[u_{\ell}, u_{r}\right](x / t)$ is a coupling solution to Riemann problem (2.1), (2.2).

- A coupling Riemann solver $\mathcal{R S}$ is coherent at $\left(u_{\ell}, u_{r}\right) \in \mathrm{D}_{\mathrm{v}}$ if $U \doteq \mathcal{R} \mathcal{S}\left[u_{\ell}, u_{r}\right]$ satisfies

$$
\mathcal{R S}\left[U\left(0^{-}\right), U\left(0^{+}\right)\right](\xi)= \begin{cases}U\left(0^{-}\right) & \text {if } \xi<0, \\ U\left(0^{+}\right) & \text {if } \xi \geq 0 .\end{cases}
$$

We say that $\mathcal{R S}$ is coherent if it is coherent in the whole of $\mathrm{D}$.

- A subset $\Omega_{u_{-}} \times \Omega_{u_{+}}, u_{ \pm} \in \Omega$, of $\mathrm{D}$ is a coupling invariant domain of $\mathcal{R S}$ if

$$
\mathcal{R S}\left[\Omega_{u_{-}}, \Omega_{u_{+}}\right]\left(0^{-}\right) \in \Omega_{-}, \quad \mathcal{R S}\left[\Omega_{u_{-}}, \Omega_{u_{+}}\right]\left(0^{+}\right) \in \Omega_{+},
$$

and for any $\xi>0$

$$
\mathcal{R S}\left[\Omega_{u_{-}}, \Omega_{u_{+}}\right](-\xi) \in \Omega_{-}, \quad \mathcal{R S}\left[\Omega_{u_{-}}, \Omega_{u_{+}}\right](\xi) \in \Omega_{+} .
$$


Condition $\left(\mathbf{c h}_{\mathbf{c}}\right)$ is the generalization of $(\mathbf{c h})$ to the case of a coupling Riemann solver. We stress that $(\mathbf{c h})_{1}$ at $x=0$ has no counterpart in $\left(\mathbf{c h}_{\mathbf{c}}\right)$ because it follows from (3.1). If $\Omega_{-}=\Omega_{+}$, then any (coherent) Riemann solver is a (coherent) coupling Riemann solver; the converse implications are not necessarily true. Indeed, the solutions corresponding to a coupling Riemann solver are not necessarily weak solutions because they may fail to satisfy the second Rankine-Hugoniot condition (2.4) at $x=0$. Notice that if $\mathrm{D}_{\mathrm{v}}$ is an invariant domain of $\mathcal{R S}$, then $(t, x) \mapsto \mathcal{R} \mathcal{S}\left[u_{\ell}, u_{r}\right](x / t)$ satisfies (3.1). At last, the product of distinct invariant domains $\Omega_{u_{-}}$and $\Omega_{u_{+}}$of the Lax Riemann-solver $\mathcal{R} \mathcal{S}_{\mathrm{p}}$ is not, in general, a coupling invariant domain of $\mathcal{R} \mathcal{S}_{\mathrm{p}}$.

We now aim at defining a general coupling Riemann solver $\mathcal{R} \mathcal{S}_{\mathrm{v}}: \mathrm{D}_{\mathrm{v}} \rightarrow \mathbf{B V}(\mathbb{R} ; \Omega)$ in presence of a valve. We denote by $A \subseteq D_{v}$ the set of Riemann data for which the valve is active; it is assigned according to the valve under consideration. The set $D_{v} \backslash A$ is the set of Riemann data for which the valve is inactive.

To any Riemann datum $\left(u_{\ell}, u_{r}\right) \in \mathrm{A}$, we assign a priori the flow $Q_{\mathrm{A}}=Q_{\mathrm{A}}\left(u_{\ell}, u_{r}\right)$ through the valve. A key point is to establish the range of values of $Q_{\mathrm{A}}$ that make the model meaningful. We discuss this important issue in the following three items.

(i) A natural requirement would be $Q_{\mathrm{A}}\left(u_{\ell}, u_{r}\right) \in\left[q\left(u_{r}\right), \bar{q}\left(u_{\ell}\right)\right]$ for any $\left(u_{\ell}, u_{r}\right) \in \mathrm{A}$. This assumption guarantees that the states $\hat{u}\left(Q_{\mathrm{A}}, u_{\ell}\right)$ and $\breve{u}\left(Q_{\mathrm{A}}, u_{r}\right)$ are well defined, but the solution of the initialboundary value problem on the left (right) may involve waves with positive (negative) speeds, which is not meaningful. To avoid this possibility we define $\bar{Q}: \Omega \rightarrow \mathbb{R}$ and $\underline{Q}: \Omega \rightarrow \mathbb{R}$ as

$$
\bar{Q}(u) \doteq\left\{\begin{array} { l l } 
{ \overline { q } ( u ) } & { \text { if } v \leqslant a , } \\
{ q } & { \text { if } v > a , }
\end{array} \quad \underline { Q } ( u ) \doteq \left\{\begin{array}{ll}
\underline{q}(u) & \text { if } v \geq-a, \\
q & \text { if } v<-a,
\end{array}\right.\right.
$$

see Figure 4. Then we strengthen the above requirement to

$$
Q_{\mathrm{A}}\left(u_{\ell}, u_{r}\right) \in\left[\underline{Q}\left(u_{r}\right), \bar{Q}\left(u_{\ell}\right)\right] .
$$

Notice that the interval in (3.5) is never empty because

$$
\underline{Q}\left(u_{r}\right)<0<\bar{Q}\left(u_{\ell}\right) .
$$

Assumption (3.5) is a causal condition: it implies that the following initial-boundary Riemann problems at $x=0$ are solved by waves of negative speeds in $x<0$ and positive speeds in $x>0$, see $[7,8]$.
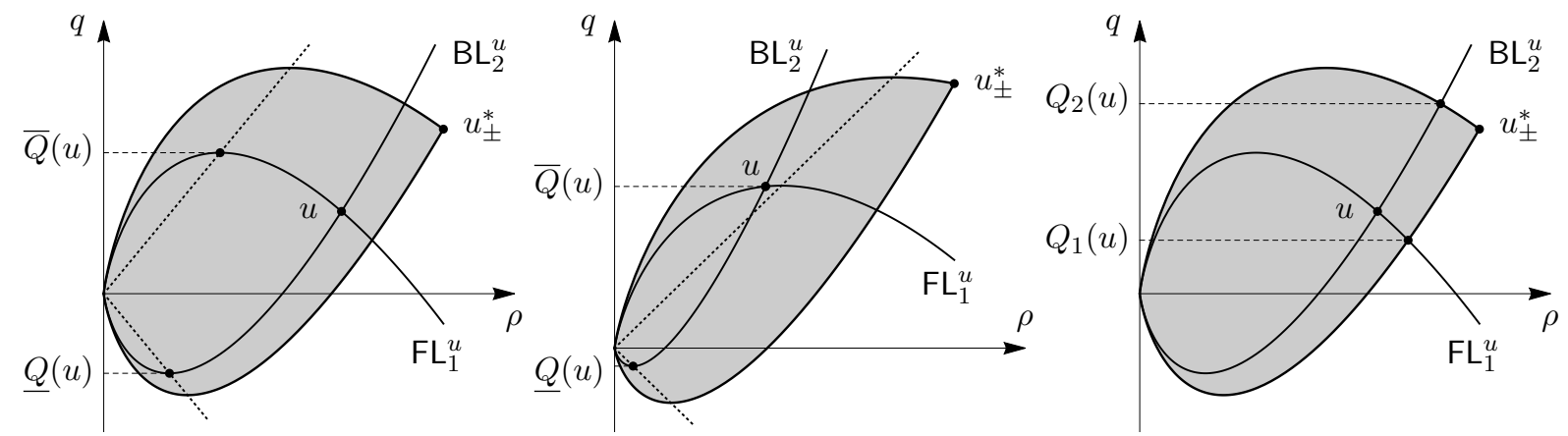

Figure 4: Geometrical meaning of $Q(u), \bar{Q}(u)$, on the left with $v<a$ and in the center with $v>a$. On the right $Q_{1}(u)$ and $Q_{2}(u)$. For simplicity we assumed $u_{-}^{*}=u_{+}^{*}$.

(ii) We implicitly define the functions $Q_{1}: \Omega_{-} \rightarrow \mathbb{R}$ and $Q_{2}: \Omega_{+} \rightarrow \mathbb{R}$ by

$$
\begin{aligned}
& Q_{1}(u) \doteq\left\{q_{o}: \exists \rho_{o} \text { s.t. } q_{o}=\mathcal{S}_{1}^{u}\left(\rho_{o}\right)=\mathcal{R}_{2}^{u_{-}^{*}}\left(\rho_{o}\right)\right\}, \\
& Q_{2}(u) \doteq\left\{q_{o}: \exists \rho_{o} \text { s.t. } q_{o}=\mathcal{S}_{2}^{u}\left(\rho_{o}\right)=\mathcal{R}_{1}^{u_{+}^{*}}\left(\rho_{o}\right)\right\},
\end{aligned}
$$


see Figure 4 on the right. Therefore $Q_{1}(u)$ is the value of the flux at the intersection of the curve $\mathrm{FL}_{1}^{u}$ with the (lower) boundary $\mathrm{R}_{2}^{u_{-}^{*}}$ of $\Omega_{-}$, while $Q_{2}(u)$ is the analogous value at the intersection of $\mathrm{BL}_{2}^{u}$ with the (upper) boundary $\mathrm{R}_{1}^{u_{+}^{*}}$ of $\Omega_{+}$. Both $Q_{1}$ and $Q_{2}$ depend on $u_{ \pm}^{*}$ but we drop this dependence. We require

$$
Q_{\mathrm{A}}\left(u_{\ell}, u_{r}\right) \in\left[Q_{1}\left(u_{\ell}\right), Q_{2}\left(u_{r}\right)\right]
$$

in order that the following coupling solutions are valued in $\Omega_{-} \times \Omega_{+}$. Notice that

$$
Q_{1}\left(u_{\ell}\right) \leqslant q_{-}^{*}, \quad Q_{2}\left(u_{r}\right) \geqslant q_{+}^{*} .
$$

However, differently from the interval $\left[\underline{Q}\left(u_{r}\right), \bar{Q}\left(u_{\ell}\right)\right]$ in $(3.5)$, the interval $\left[Q_{1}\left(u_{\ell}\right), Q_{2}\left(u_{r}\right)\right]$ may be empty if either $q_{-}^{*}>0$ or $q_{+}^{*}<0$. For example, consider the case $q_{-}^{*}>0>q_{+}^{*}$ and refer to Figure 5: there are states $\left(u_{\ell}, u_{r}\right) \in \mathrm{D}_{\mathrm{v}}$ such that $Q_{2}\left(u_{r}\right)<0<Q_{1}\left(u_{\ell}\right)$. It is possible to show that the interval $\left[Q_{1}\left(u_{\ell}\right), Q_{2}\left(u_{r}\right)\right]$ is not empty under suitable technical assumptions on the set $\mathrm{A}$; we do not consider such conditions here. For simplicity we assume

$$
q_{-}^{*}=0=q_{+}^{*} .
$$

Condition (H.1) ensures $0 \in\left[Q_{1}\left(u_{\ell}\right), Q_{2}\left(u_{r}\right)\right]$ by $(3.8)$, hence $\left[Q_{1}\left(u_{\ell}\right), Q_{2}\left(u_{r}\right)\right] \neq \emptyset$ for $\left(u_{\ell}, u_{r}\right) \in \mathrm{D}_{\mathrm{v}}$.
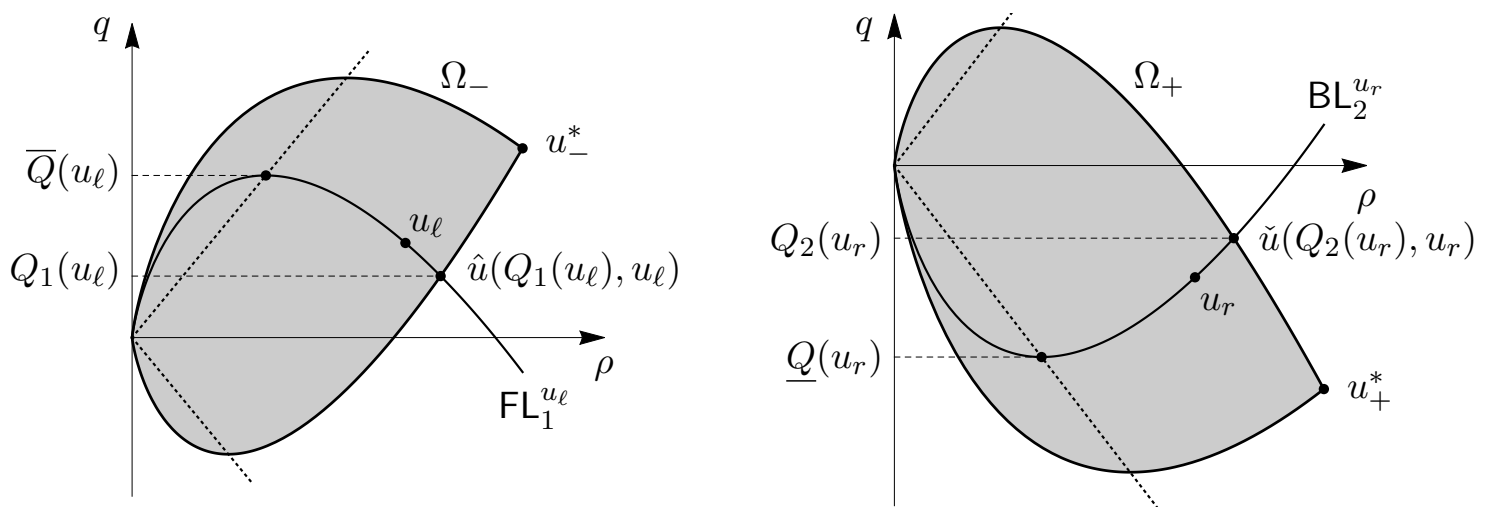

Figure 5: Left: the case $q_{-}^{*}>0$. Right: the case $q_{+}^{*}<0$.

(iii) We denote

$$
Q^{\min }\left(u_{\ell}, u_{r}\right) \doteq \max \left\{\underline{Q}\left(u_{r}\right), Q_{1}\left(u_{\ell}\right)\right\}, \quad Q^{\max }\left(u_{\ell}, u_{r}\right) \doteq \min \left\{\bar{Q}\left(u_{\ell}\right), Q_{2}\left(u_{r}\right)\right\} .
$$

Notice that by (H.1), (3.6) and (3.8) we deduce

$$
Q^{\min }\left(u_{\ell}, u_{r}\right) \leqslant 0 \leqslant Q^{\max }\left(u_{\ell}, u_{r}\right) .
$$

Then we define

$$
I_{\mathrm{A}}\left(u_{\ell}, u_{r}\right) \doteq\left[Q^{\min }\left(u_{\ell}, u_{r}\right), Q^{\max }\left(u_{\ell}, u_{r}\right)\right]=\left[\underline{Q}\left(u_{r}\right), \bar{Q}\left(u_{\ell}\right)\right] \cap\left[Q_{1}\left(u_{\ell}\right), Q_{2}\left(u_{r}\right)\right] .
$$

In this way we can prescribe the admissible range of values of $Q_{\mathrm{A}}$ : namely, we choose

$$
Q_{\mathrm{A}}\left(u_{\ell}, u_{r}\right) \in I_{\mathrm{A}}\left(u_{\ell}, u_{r}\right) \quad \text { if }\left(u_{\ell}, u_{r}\right) \in \mathrm{A} .
$$

Under $(\mathbf{H . 1})$, we have $0 \in I_{\mathrm{A}}\left(u_{\ell}, u_{r}\right) \neq \emptyset$ for any $\left(u_{\ell}, u_{r}\right) \in \mathrm{A}$, and then a choice of $Q_{\mathrm{A}}\left(u_{\ell}, u_{r}\right)$ satisfying $(\mathbf{H . 2})$ is possible. As a consequence, by (H.2) the states

$$
\hat{u}_{\mathrm{A}}\left(u_{\ell}, u_{r}\right) \doteq \hat{u}\left(Q_{\mathrm{A}}\left(u_{\ell}, u_{r}\right), u_{\ell}\right), \quad \check{u}_{\mathrm{A}}\left(u_{\ell}, u_{r}\right) \doteq \check{u}\left(Q_{\mathrm{A}}\left(u_{\ell}, u_{r}\right), u_{r}\right),
$$

are well defined for any $\left(u_{\ell}, u_{r}\right) \in \mathrm{A}$. Notice that

$$
\hat{q}_{\mathrm{A}}\left(u_{\ell}, u_{r}\right)=Q_{\mathrm{A}}\left(u_{\ell}, u_{r}\right)=\check{q}_{\mathrm{A}}\left(u_{\ell}, u_{r}\right) \quad \text { for any }\left(u_{\ell}, u_{r}\right) \in \mathrm{A} .
$$


We can now give the definition of the solver $\mathcal{R S}_{\mathrm{v}}$.

Definition 3.3. Assume (H.1). Consider $\mathrm{A} \subseteq \mathrm{D}_{\mathrm{v}}$ and a function $Q_{\mathrm{A}}: \mathrm{A} \rightarrow \mathbb{R}$ satisfying $(\mathbf{H . 2})$. We define $\mathcal{R S}_{\mathrm{v}}: \mathrm{D}_{\mathrm{v}} \rightarrow \mathbf{B V}(\mathbb{R} ; \Omega)$ as

$$
\begin{aligned}
& \mathcal{R S}_{\mathrm{v}}\left[u_{\ell}, u_{r}\right](\xi) \doteq\left\{\begin{array}{l}
\mathcal{R} \mathcal{S}_{\mathrm{p}}\left[u_{\ell}, \hat{u}_{\mathrm{A}}\left(u_{\ell}, u_{r}\right)\right](\xi) \quad \text { if } \xi<0, \\
\mathcal{R} \mathcal{S}_{\mathrm{p}}\left[\check{u}_{\mathrm{A}}\left(u_{\ell}, u_{r}\right), u_{r}\right](\xi) \quad \text { if } \xi \geq 0,
\end{array} \quad \text { if }\left(u_{\ell}, u_{r}\right) \in \mathrm{A},\right. \\
& \mathcal{R S}_{\mathrm{v}}\left[u_{\ell}, u_{r}\right] \doteq \mathcal{R} \mathcal{S}_{\mathrm{p}}\left[u_{\ell}, u_{r}\right], \quad \text { if }\left(u_{\ell}, u_{r}\right) \in \mathrm{D}_{\mathrm{v}} \backslash \mathrm{A} .
\end{aligned}
$$

For $x \in \mathbb{R}$ and $t \in \mathbb{R}_{+}$we denote

$$
u_{\mathrm{v}}(t, x) \doteq \mathcal{R S}_{\mathrm{v}}\left[u_{\ell}, u_{r}\right](x / t) \quad \text { and } \quad u_{\mathrm{v}}^{ \pm} \doteq u_{\mathrm{v}}\left(t, 0^{ \pm}\right) .
$$

Lemma 3.4. For any $\left(u_{\ell}, u_{r}\right) \in \mathrm{A}$ we have

$$
\begin{aligned}
& u_{\mathrm{v}}^{-}= \begin{cases}u_{\ell} \neq \hat{u}_{\mathrm{A}}\left(u_{\ell}, u_{r}\right) & \text { if } v_{\ell}>a \text { and } Q_{\mathrm{A}}\left(u_{\ell}, u_{r}\right)=q_{\ell}, \\
\hat{u}_{\mathrm{A}}\left(u_{\ell}, u_{r}\right) & \text { otherwise, }\end{cases} \\
& u_{\mathrm{v}}^{+}= \begin{cases}u_{r} \neq \check{u}_{\mathrm{A}}\left(u_{\ell}, u_{r}\right) & \text { if } v_{r}<-a \text { and } Q_{\mathrm{A}}\left(u_{\ell}, u_{r}\right)=q_{r}, \\
\check{u}_{\mathrm{A}}\left(u_{\ell}, u_{r}\right) & \text { otherwise. }\end{cases}
\end{aligned}
$$

Proof. About (3.15) we have some cases.

(i) If $v_{\ell}>a$, then $\rho_{\ell}<\bar{\rho}\left(u_{\ell}\right)$ by Lemma 2.4 and $\bar{Q}\left(u_{\ell}\right)=q_{\ell}$ by $(3.4)_{1}$. Therefore $Q_{\mathrm{A}}\left(u_{\ell}, u_{r}\right) \leqslant q_{\ell}$.

If $Q_{\mathrm{A}}\left(u_{\ell}, u_{r}\right)=q_{\ell}$, then in the $u$-plane the horizontal line through $u_{\ell}$ meets the curve $\mathrm{FL}_{1}^{u_{\ell}}$ at the point $\hat{u}_{\mathrm{A}}\left(u_{\ell}, u_{r}\right)$ with $\rho_{\ell}<\bar{\rho}\left(u_{\ell}\right)<\hat{\rho}_{\mathrm{A}}\left(u_{\ell}, u_{r}\right)$. Then $u_{\ell} \neq \hat{u}_{\mathrm{A}}\left(u_{\ell}, u_{r}\right)$. Moreover, $u_{\ell}$ is connected to $\hat{u}_{\mathrm{A}}\left(u_{\ell}, u_{r}\right)$ by a stationary 1-shock and so $u_{\mathrm{v}}^{-}=u_{\ell}$.

If $Q_{\mathrm{A}}\left(u_{\ell}, u_{r}\right)<q_{\ell}$, then $u_{\ell}$ is connected to $\hat{u}_{\mathrm{A}}\left(u_{\ell}, u_{r}\right)$ by a 1 -shock with negative speed and so $u_{\mathrm{v}}^{-}=\hat{u}_{\mathrm{A}}\left(u_{\ell}, u_{r}\right)$.

(ii) If $v_{\ell} \leq a$, then $\rho_{\ell} \geqslant \bar{\rho}\left(u_{\ell}\right)$ and $\bar{Q}\left(u_{\ell}\right)=\bar{q}\left(u_{\ell}\right)$. Therefore $Q_{\mathrm{A}}\left(u_{\ell}, u_{r}\right) \leqslant \bar{q}\left(u_{\ell}\right)$ and $u_{\ell}$ is connected to $\hat{u}_{\mathrm{A}}\left(u_{\ell}, u_{r}\right)$ by a possible null 1-wave contained in $x \leqslant 0$ and then $u_{\mathrm{v}}^{-}=\hat{u}_{\mathrm{A}}\left(u_{\ell}, u_{r}\right)$.

Identity (3.16) is proved analogously.

As a consequence of Lemma 3.4 and (3.11) we have

$$
q_{\mathrm{v}}^{-}=q_{\mathrm{v}}^{+}=Q_{\mathrm{A}}\left(u_{\ell}, u_{r}\right) \quad \text { for any }\left(u_{\ell}, u_{r}\right) \in \mathrm{A} .
$$

If $\rho_{-}^{*}=\rho_{+}^{*}$ then $(\mathbf{H . 1})$ implies $\Omega_{-}=\Omega_{+}$. In this case the restriction of $\mathcal{R} \mathcal{S}_{\mathrm{p}}$ to $\mathrm{D}_{\mathrm{v}}$ is a coupling Riemann solver and there is actually no need to activate the valve. We assume therefore $\rho_{-}^{*} \neq \rho_{+}^{*}$ from now on and focus for simplicity on the case

$$
\rho_{-}^{*}<\rho_{+}^{*} .
$$

Then we have $\Omega_{-} \subsetneq \Omega_{+}$. Therefore $u_{\mathrm{p}}$ takes values in $\Omega_{+}$for any $\left(u_{\ell}, u_{r}\right) \in \mathrm{D}_{\mathrm{v}} \subset \Omega_{+} \times \Omega_{+}$; however, the restriction of $u_{\mathrm{p}}$ to $x<0$ is not necessarily valued in $\Omega_{-}$and then $\mathcal{R} \mathcal{S}_{\mathrm{p}}: \mathrm{D}_{\mathrm{v}} \rightarrow \mathbf{B V}(\mathbb{R} ; \Omega)$ is not a coupling Riemann solver. For this reason we define the sets

$$
\begin{aligned}
& \mathrm{A}_{1} \doteq\left\{\left(u_{\ell}, u_{r}\right) \in \mathrm{D}_{\mathrm{v}}: u_{r} \in \Omega_{+} \backslash \Omega_{-}, \widetilde{u}\left(u_{\ell}, u_{r}\right) \in \Omega_{+} \backslash \Omega_{-}\right\} \\
& \mathrm{A}_{2} \doteq\left\{\left(u_{\ell}, u_{r}\right) \in \mathrm{D}_{\mathrm{v}}: u_{r} \in \Omega_{+} \backslash \Omega_{-}, \widetilde{u}\left(u_{\ell}, u_{r}\right) \in \Omega_{-}, \rho_{r}<\widetilde{\rho}\left(u_{\ell}, u_{r}\right), q_{r}>\widetilde{q}\left(u_{\ell}, u_{r}\right)\right\}
\end{aligned}
$$

Notice that $\left(u_{\ell}, u_{r}\right) \in \mathrm{A}_{1}$ if and only if $\mathcal{R S}_{\mathrm{p}}\left[u_{\ell}, \widetilde{u}\left(u_{\ell}, u_{r}\right)\right]$ is a 1-shock with negative speed but $\widetilde{u}\left(u_{\ell}, u_{r}\right)$ does not belong to $\Omega_{-}$, see Figure 6 on the left. On the other hand, we have $\left(u_{\ell}, u_{r}\right) \in \mathrm{A}_{2}$ if and only if $\widetilde{u}\left(u_{\ell}, u_{r}\right)$ belongs to $\Omega_{-}$, but $\mathcal{R} \mathcal{S}_{\mathrm{p}}\left[\widetilde{u}\left(u_{\ell}, u_{r}\right), u_{r}\right]$ is a 2 -shock with negative speed and $u_{r} \in \Omega_{+} \backslash \Omega_{-}$, see Figure 6 on the right. In both cases the restriction of $u_{\mathrm{p}}$ to $x<0$ does not take values in $\Omega_{-}$and fails to satisfy (3.1). The next proposition shows that also the converse is true. We denote

$$
A_{0} \doteq A_{1} \cup A_{2} .
$$



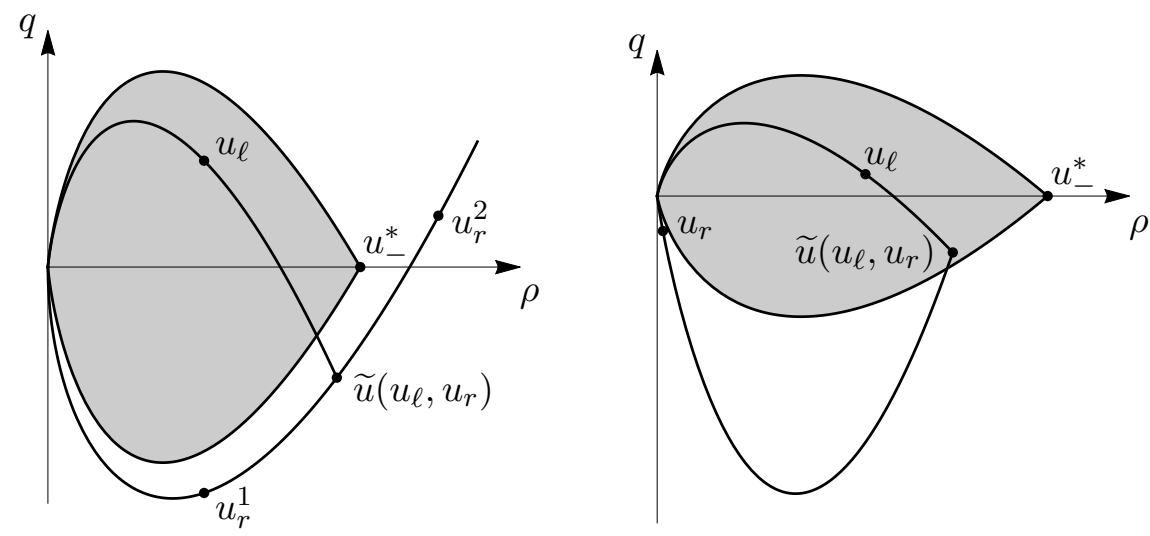

Figure 6: On the left, $\left(u_{\ell}, u_{r}\right) \in \mathrm{A}_{1}$. Here $u_{r}^{1}$ and $u_{r}^{2}$ represent the right state $u_{r}$ in two different cases: $\mathcal{R} \mathcal{S}_{\mathrm{p}}\left[\widetilde{u}\left(u_{\ell}, u_{r}\right), u_{r}\right]$ is a 2-shock or a 2-rarefaction, respectively. On the right, $\left(u_{\ell}, u_{r}\right) \in \mathrm{A}_{2}$. The shaded regions correspond to $\Omega_{-}$.

Proposition 3.5. We have that $u_{\mathrm{p}}$ fails to satisfy (3.1) (in $\mathbb{R}_{-}$) if and only if $\left(u_{\ell}, u_{r}\right) \in \mathrm{A}_{0}$.

The proof is deferred to Subsection 5.1. Notice that $A_{0} \neq D_{v}$ because, for instance, we have

$$
\Omega_{-} \times \Omega_{-} \subset \mathrm{D}_{\mathrm{v}} \backslash \mathrm{A}_{0}
$$

Since by Proposition 3.5 we saw that $u_{\mathrm{p}}$ fails to satisfy (3.1) if and only if $\left(u_{\ell}, u_{r}\right) \in \mathrm{A}_{0}$, we assume that the valve is active in correspondence of the states in $A_{0}$; then, we assume

$$
A \supseteq A_{0}
$$

The next proposition shows that $\mathcal{R S}_{\mathrm{v}}$ is a coupling Riemann solver under the hypotheses $(\mathbf{H . 1})-($ H.4) . These conditions are always assumed from now on without any further mention.

Proposition 3.6. $\mathcal{R S}_{\mathrm{v}}: \mathrm{D}_{\mathrm{v}} \rightarrow \mathbf{B V}(\mathbb{R} ; \Omega)$ is a coupling Riemann solver.

The proof is deferred to Subsection 5.2. In particular, Proposition 3.6 and (3.3) imply that

$$
q_{\mathrm{v}}^{-}=q_{\mathrm{v}}^{+} \quad \text { for any }\left(u_{\ell}, u_{r}\right) \in \mathrm{D}_{\mathrm{v}} .
$$

Now, our main concern is to investigate the coherence of coupling Riemann solvers $\mathcal{R S}_{\mathrm{v}}$. Notice that by Definition 3.2 this amounts to require $\left(\mathbf{c h}_{\mathbf{c}}\right)$, which becomes, by using the notation in (3.14),

$$
\mathcal{R S}_{\mathrm{v}}\left[u_{\mathrm{v}}^{-}, u_{\mathrm{v}}^{+}\right](\xi)= \begin{cases}u_{\mathrm{v}}^{-} & \text {if } \xi<0, \\ u_{\mathrm{v}}^{+} & \text {if } \xi \geq 0 .\end{cases}
$$

\section{Applications}

In the previous section we modeled the gas flow through a valve by prescribing, in particular, that it took place in invariant domains. This requirement alone cannot single out a solution to the Riemann problem; to this aim, in this section we introduce a general model of valve that maximizes the throughput to allow for the highest possible volume flow. More precisely, we define

$$
Q_{\mathrm{A}}\left(u_{\ell}, u_{r}\right) \doteq Q^{\max }\left(u_{\ell}, u_{r}\right),
$$

for $\left(u_{\ell}, u_{r}\right)$ in some set $\mathrm{A}$ to be specified. We observe that

$$
Q_{\mathrm{A}}\left(u_{\ell}, u_{r}\right) \geqslant 0
$$

by (3.9), with equality if and only if $u_{r}=u_{+}^{*}$. As a consequence, the flow is positive or null as long as the valve is active. On the other hand, if the valve is not active, then the flow can be negative. Moreover, from (4.1) and (3.16) it follows

$$
u_{\mathrm{v}}^{+}=\check{u}_{\mathrm{A}}\left(u_{\ell}, u_{r}\right) \quad \text { for any }\left(u_{\ell}, u_{r}\right) \in \mathrm{A} .
$$




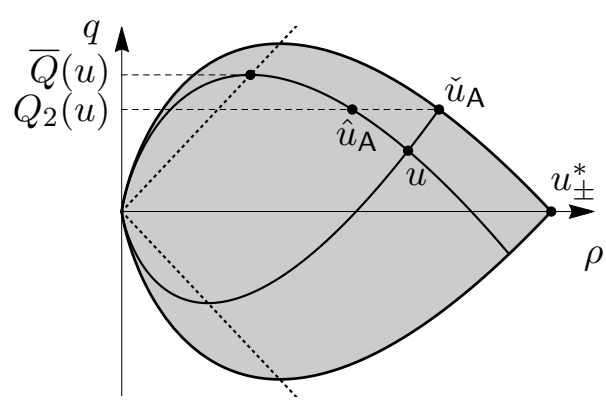

Figure 7: By (4.1), if $\left(u_{\ell}, u_{r}\right) \doteq(u, u)$ with $u$ as in this picture, then $q_{\mathrm{v}}^{ \pm}=Q_{2}(u)>q_{\mathrm{p}}^{ \pm}=q$. Above, $\hat{u}_{\mathrm{A}}$ and $\check{u}_{\mathrm{A}}$ stand for $\hat{u}_{\mathrm{A}}(u, u)$ and $\check{u}_{\mathrm{A}}(u, u)$, respectively.
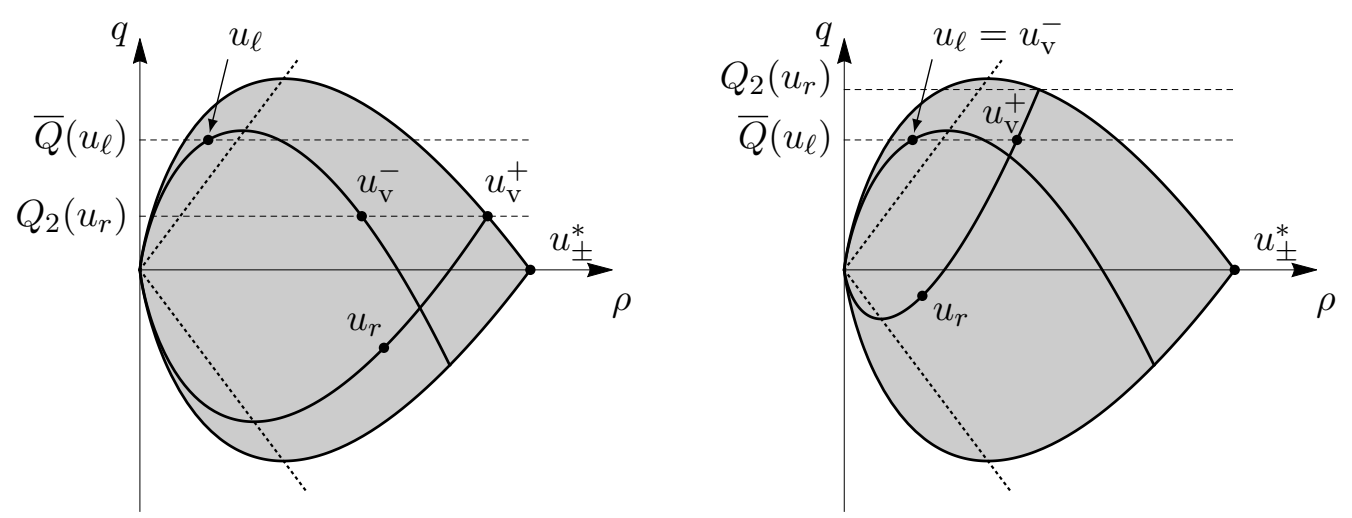

Figure 8: Cases (c.1) and (c.3) in the proof of Lemma 4.1, both of them in subcase $v_{\ell}>a$.

For simplicity we take $u_{-}^{*}=u_{+}^{*}$.

We stress that $q_{\mathrm{v}}^{ \pm} \geqslant q_{\mathrm{p}}^{ \pm}$by the choice (4.1) for any $\left(u_{\ell}, u_{r}\right) \in \mathrm{D}_{\mathrm{v}}$, see for instance Figure 7 with $u_{\ell}=u_{r}$.

The next lemma shows that the choice (4.1) ensures a kind of stability for the traces. This property ensures the coherence as long as the valve is active.

Lemma 4.1. If both $\left(u_{\ell}, u_{r}\right)$ and $\left(u_{\mathrm{v}}^{-}, u_{\mathrm{v}}^{+}\right)$belong to $\mathrm{A}$, then

$$
Q_{\mathrm{A}}\left(u_{\mathrm{v}}^{-}, u_{\mathrm{v}}^{+}\right)=q_{\mathrm{v}}^{ \pm}=Q_{\mathrm{A}}\left(u_{\ell}, u_{r}\right) .
$$

Proof. By (3.17) we have $q_{\mathrm{v}}^{ \pm}=Q_{\mathrm{A}}\left(u_{\ell}, u_{r}\right)$. To complete the proof it remains to prove that $Q_{\mathrm{A}}\left(u_{\mathrm{v}}^{-}, u_{\mathrm{v}}^{+}\right)=q_{\mathrm{v}}^{ \pm}$. We use many times (4.1), (4.3) and Lemma 3.4. We distinguish three main cases, see Figure 8.

(c.1) Assume $Q_{\mathrm{A}}\left(u_{\ell}, u_{r}\right)=Q_{2}\left(u_{r}\right) \leqslant \bar{Q}\left(u_{\ell}\right)$. Since $Q_{\mathrm{A}}\left(u_{\ell}, u_{r}\right)=Q_{2}\left(u_{r}\right)$, by $(4.3)$ we have $u_{\mathrm{v}}^{+}=\check{u}_{\mathrm{A}}\left(u_{\ell}, u_{r}\right) \in$ $\mathrm{R}_{1}^{u_{+}^{*}}$ and therefore $Q_{2}\left(u_{\mathrm{v}}^{+}\right)=q_{\mathrm{v}}^{+}$. By $(3.4)_{1}$ we deduce $\bar{Q}\left(u_{\mathrm{v}}^{-}\right) \geqslant q_{\mathrm{v}}^{-}$. As a consequence $Q_{2}\left(u_{\mathrm{v}}^{+}\right)=q_{\mathrm{v}}^{ \pm} \leqslant$ $\bar{Q}\left(u_{\mathrm{v}}^{-}\right)$and then $Q_{\mathrm{A}}\left(u_{\mathrm{v}}^{-}, u_{\mathrm{v}}^{+}\right)=Q_{2}\left(u_{\mathrm{v}}^{+}\right)=q_{\mathrm{v}}^{ \pm}$.

(c.2) Assume $Q_{\mathrm{A}}\left(u_{\ell}, u_{r}\right)=\bar{Q}\left(u_{\ell}\right)<Q_{2}\left(u_{r}\right)$ and $v_{\ell} \leqslant a$. Since $Q_{\mathrm{A}}\left(u_{\ell}, u_{r}\right)<Q_{2}\left(u_{r}\right)$, we have $u_{\mathrm{v}}^{+} \notin \mathrm{R}_{1}^{u_{+}^{*}}$ and therefore $Q_{2}\left(u_{\mathrm{v}}^{+}\right)>q_{\mathrm{v}}^{+}$. Since $v_{\ell} \leqslant a$, we infer $Q_{\mathrm{A}}\left(u_{\ell}, u_{r}\right)=\bar{Q}\left(u_{\ell}\right)=\bar{q}\left(u_{\ell}\right)$. Hence by $(3.15)$ we obtain $u_{\mathrm{v}}^{-}=\bar{u}\left(u_{\ell}\right)$; then $v_{\mathrm{v}}^{-}=\bar{v}\left(u_{\ell}\right)=a$ and by $(3.4)_{1}$ we deduce $\bar{Q}\left(u_{\mathrm{v}}^{-}\right)=q_{\mathrm{v}}^{-}$. As a consequence, $\bar{Q}\left(u_{\mathrm{v}}^{-}\right)=q_{\mathrm{v}}^{ \pm}<Q_{2}\left(u_{\mathrm{v}}^{+}\right)$and at last this yields $Q_{\mathrm{A}}\left(u_{\mathrm{v}}^{-}, u_{\mathrm{v}}^{+}\right)=\bar{Q}\left(u_{\mathrm{v}}^{-}\right)=q_{\mathrm{v}}^{ \pm}$.

(c.3) Assume $Q_{\mathrm{A}}\left(u_{\ell}, u_{r}\right)=\bar{Q}\left(u_{\ell}\right)<Q_{2}\left(u_{r}\right)$ and $v_{\ell}>a$. Since $Q_{\mathrm{A}}\left(u_{\ell}, u_{r}\right)<Q_{2}\left(u_{r}\right)$, we have $u_{\mathrm{v}}^{+} \notin \mathrm{R}_{1}^{u_{+}^{*}}$ and then $Q_{2}\left(u_{\mathrm{v}}^{+}\right)>q_{\mathrm{v}}^{+}$. Since $v_{\ell}>a$, we deduce $Q_{\mathrm{A}}\left(u_{\ell}, u_{r}\right)=\bar{Q}\left(u_{\ell}\right)=q_{\ell}$. Hence by $(3.15)$ we infer $u_{\mathrm{v}}^{-}=$ $u_{\ell}$. As a consequence, $\bar{Q}\left(u_{\mathrm{v}}^{-}\right)=\bar{Q}\left(u_{\ell}\right)=q_{\ell}=q_{\mathrm{v}}^{ \pm}<Q_{2}\left(u_{\mathrm{v}}^{+}\right)$and this gives $Q_{\mathrm{A}}\left(u_{\mathrm{v}}^{-}, u_{\mathrm{v}}^{+}\right)=\bar{Q}\left(u_{\mathrm{v}}^{-}\right)=q_{\mathrm{v}}^{ \pm}$.

This concludes the proof.

Proposition 4.2. The coupling Riemann solver $\mathcal{R} \mathcal{S}_{\mathrm{v}}$ is coherent if for any $\left(u_{\ell}, u_{r}\right) \in \mathrm{D}_{\mathrm{v}}$ we have

$$
\left(u_{\ell}, u_{r}\right) \in \mathrm{A} \Longleftrightarrow\left(u_{\mathrm{v}}^{-}, u_{\mathrm{v}}^{+}\right) \in \mathrm{A} .
$$


Proof. We have to show that $\left(\mathbf{c h}_{\mathbf{v}}\right)$ is satisfied for any $\left(u_{\ell}, u_{r}\right) \in \mathrm{D}_{\mathrm{v}}$ under condition (4.4). Assume that $\left(u_{\ell}, u_{r}\right) \in \mathrm{A}$. By $(4.4)$ we have $\left(u_{\mathrm{v}}^{-}, u_{\mathrm{v}}^{+}\right) \in \mathrm{A}$. Therefore by (3.11) and Lemma 4.1 we have

$$
\hat{q}_{\mathrm{A}}\left(u_{\mathrm{v}}^{-}, u_{\mathrm{v}}^{+}\right)=\check{q}_{\mathrm{A}}\left(u_{\mathrm{v}}^{-}, u_{\mathrm{v}}^{+}\right)=Q_{\mathrm{A}}\left(u_{\mathrm{v}}^{-}, u_{\mathrm{v}}^{+}\right)=q_{\mathrm{v}}^{ \pm} .
$$

Furthermore, since $Q_{\mathrm{A}}\left(u_{\mathrm{v}}^{-}, u_{\mathrm{v}}^{+}\right) \geqslant 0$ by (4.2), we have that $\mathcal{R} \mathcal{S}_{\mathrm{p}}\left[u_{\mathrm{v}}^{-}, \hat{u}_{\mathrm{A}}\left(u_{\mathrm{v}}^{-}, u_{\mathrm{v}}^{+}\right)\right]$is either constant (if $\left.u_{\mathrm{v}}^{-}=\hat{u}_{\mathrm{A}}\left(u_{\mathrm{v}}^{-}, u_{\mathrm{v}}^{+}\right)\right)$or has a stationary 1-shock (if $u_{\mathrm{v}}^{-} \neq \hat{u}_{\mathrm{A}}\left(u_{\mathrm{v}}^{-}, u_{\mathrm{v}}^{+}\right)$, see however Subsection 5.3), while $\mathcal{R} \mathcal{S}_{\mathrm{p}}\left[\check{u}_{\mathrm{A}}\left(u_{\mathrm{v}}^{-}, u_{\mathrm{v}}^{+}\right), u_{\mathrm{v}}^{+}\right] \equiv u_{\mathrm{v}}^{+}$. As a consequence $(3.12)$ yields

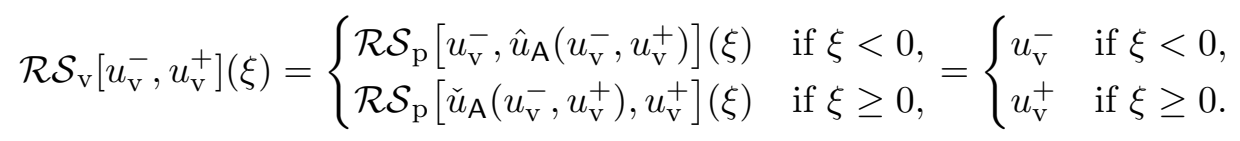

This proves the coherence in the case $\left(u_{\ell}, u_{r}\right) \in \mathrm{A}$. If $\left(u_{\ell}, u_{r}\right) \in \mathrm{D}_{\mathrm{v}} \backslash \mathrm{A}$, then by (4.4) it is sufficient to remind that $\mathcal{R} \mathcal{S}_{\mathrm{p}}$ is coherent.

We stress that (4.4) is satisfied if and only if both $A$ and $D_{v} \backslash A$ are coupling invariant domains.

The three coupling Riemann solvers considered in the following subsections are characterized by the same choice of $Q_{\mathrm{A}}$, see (4.1), but differ for the choice of the set A. In Subsection 4.1 we consider the "maximal" (with respect to inclusion) choice $\mathrm{A} \doteq \mathrm{D}_{\mathrm{v}}$ and prove that the corresponding $\mathcal{R S}_{\mathrm{v}}$ is coherent. On the contrary, in Subsection 4.2 we deal with the "minimal" possible choice $\mathrm{A} \doteq \mathrm{A}_{0}$ and prove that $\mathcal{R} \mathcal{S}_{\mathrm{v}}$ is not coherent. In Subsection 4.3 we investigate the existence of an intermediate set $A$, with $A_{0} \subsetneq A \subsetneq D_{v}$, such that the corresponding $\mathcal{R} \mathcal{S}_{\mathrm{v}}$ is coherent. The construction of this set is explicit and obtained by a limiting process.

\subsection{The case $A \doteq D_{v}$}

In this subsection we consider a valve that is always active and maximizes the flow. Such valve corresponds to the coupling Riemann solver $\mathcal{R S}_{\mathrm{v}}$ defined by Definition 3.3 with $Q_{\mathrm{A}}$ given by (4.1) and

$$
\mathrm{A} \doteq \mathrm{D}_{\mathrm{v}}
$$

It is a one-way valve because $Q_{\mathrm{A}}\left(u_{\ell}, u_{r}\right) \geqslant 0$ by (4.2).

Theorem 4.3 (Coherence). Under (4.5), the coupling Riemann solver $\mathcal{R S}_{\mathrm{v}}$ is coherent.

Proof. By Proposition 3.6 we have that $\mathcal{R} \mathcal{S}_{\mathrm{v}}$ is a coupling Riemann solver; hence $\mathrm{D}_{\mathrm{v}}$ is a coupling invariant domain and (4.4) is satisfied. Then it is sufficient to apply Proposition 4.2 to conclude the proof.

\subsection{The case $A \doteq A_{0}$}

In this subsection we study a valve that is active only when it is "strictly necessary" and in such a case it maximizes the flow. Such valve corresponds to the choice

$$
A \doteq A_{0}
$$

It is not a one-way valve.

Theorem 4.4 (Incoherence). Under (4.6), the coupling Riemann solver $\mathcal{R S}_{\mathrm{v}}$ is not coherent.

Proof. It is sufficient to show that there exists $\left(u_{\ell}, u_{r}\right) \in \mathrm{A}_{0}$ such that

$$
u_{\mathrm{v}}^{+} \in \Omega_{-}, \quad \mathcal{R S}_{\mathrm{p}}\left[u_{\mathrm{v}}^{-}, u_{\mathrm{v}}^{+}\right](\xi) \neq \begin{cases}u_{\mathrm{v}}^{-} & \text {if } \xi<0, \\ u_{\mathrm{v}}^{+} & \text {if } \xi \geqslant 0 .\end{cases}
$$

In fact, by $(4.7)_{1}$ we have $\left(u_{\mathrm{v}}^{-}, u_{\mathrm{v}}^{+}\right) \in \Omega_{-} \times \Omega_{-} \subseteq \mathrm{D}_{\mathrm{v}} \backslash \mathrm{A}_{0}$, see (3.21). Therefore, while the valve is active for $\left(u_{\ell}, u_{r}\right)$, by $(4.6)$ it is inactive for $\left(u_{\mathrm{v}}^{-}, u_{\mathrm{v}}^{+}\right)$and consequently $\mathcal{R} \mathcal{S}_{\mathrm{v}}\left[u_{\mathrm{v}}^{-}, u_{\mathrm{v}}^{+}\right] \equiv \mathcal{R} \mathcal{S}_{\mathrm{p}}\left[u_{\mathrm{v}}^{-}, u_{\mathrm{v}}^{+}\right]$. By $(4.7)_{2}$ we deduce the incoherence of $\mathcal{R} \mathcal{S}_{\mathrm{v}}$ at $\left(u_{\ell}, u_{r}\right)$, because

$$
\mathcal{R S}_{\mathrm{v}}\left[u_{\mathrm{v}}^{-}, u_{\mathrm{v}}^{+}\right](\xi)=\mathcal{R} \mathcal{S}_{\mathrm{p}}\left[u_{\mathrm{v}}^{-}, u_{\mathrm{v}}^{+}\right](\xi) \neq \begin{cases}u_{\mathrm{v}}^{-} & \text {if } \xi<0 \\ u_{\mathrm{v}}^{+} & \text {if } \xi \geqslant 0 .\end{cases}
$$




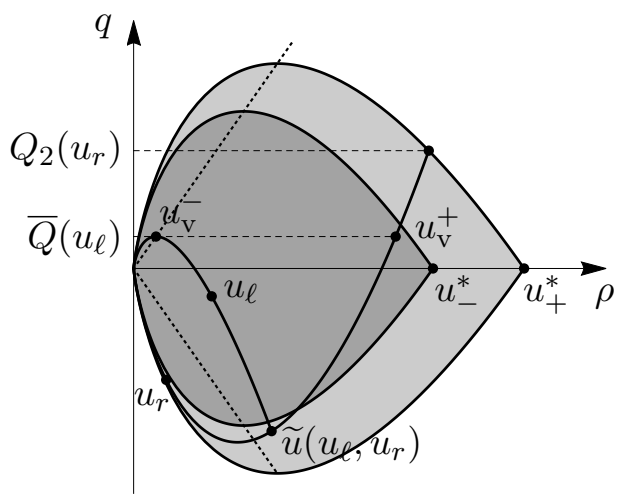

Figure 9: An example of incoherence for $\mathcal{R} \mathcal{S}_{\mathrm{v}}$ considered in Subsection 4.2. The darker shaded region represents $\Omega_{-}$, the lighter one is $\Omega_{+}$.

Indeed, condition $(4.7)_{2}$ is equivalent to require either $u_{\mathrm{v}}^{+} \notin \mathrm{FL}_{1}^{u_{\mathrm{v}}^{-}}$if $\rho_{\mathrm{v}}^{+}>\rho_{\mathrm{v}}^{-}$, or $u_{\mathrm{v}}^{-} \notin \mathrm{BL}_{2}^{u_{\mathrm{v}}^{+}}$if $\rho_{\mathrm{v}}^{-}>\rho_{\mathrm{v}}^{+}$. In other words, $\mathcal{R S}_{\mathrm{p}}\left[u_{\mathrm{v}}^{-}, u_{\mathrm{v}}^{+}\right]$is neither a stationary 1-shock, nor a stationary 2-shock. We stress that $(4.7)_{2}$ does not follow by simply assuming $u_{\mathrm{v}}^{-} \neq u_{\mathrm{v}}^{+}$and $q_{\mathrm{v}}^{-}=q_{\mathrm{v}}^{+}$.

For instance, see Figure 9, the choice

$$
a=1, \quad \rho_{\ell}=6, \quad q_{\ell}=-3 / 2, \quad \rho_{r}=5 / 2, \quad q_{r}=-6, \quad \rho_{-}^{*}=23, \quad \rho_{+}^{*}=30,
$$

satisfies (4.7). In this case $\widetilde{u}\left(u_{\ell}, u_{r}\right) \approx(10.60,-8.76) \in \Omega_{+} \backslash \Omega_{-}$and then $\left(u_{\ell}, u_{r}\right) \in \mathrm{A}_{1}$. Moreover, $Q_{2}\left(u_{r}\right) \approx$ 6.35 and $Q_{\mathrm{A}}\left(u_{\ell}, u_{r}\right)=Q^{\max }\left(u_{\ell}, u_{r}\right)=\bar{Q}\left(u_{\ell}\right)=\bar{q}\left(u_{\ell}\right) \approx 1.72$. At last $u_{\mathrm{v}}^{-}=\hat{u}_{\mathrm{A}}\left(u_{\ell}, u_{r}\right) \approx(1.72,1.72) \in \Omega_{-}$ and $u_{\mathrm{v}}^{+}=\check{u}_{\mathrm{A}}\left(u_{\ell}, u_{r}\right) \approx(20.13,1.72) \in \Omega_{-}$.

Remark 4.5. We showed in the proof of Theorem 4.4 that condition (4.7) is sufficient for the incoherence of $\mathcal{R} \mathcal{S}_{\mathrm{v}}$. However, such a condition is not necessary, in general.

\subsection{The case of the minimal set $A$ for coherence}

In Subsection 4.2 we showed that the minimal choice $A=A_{0}$ does not lead to a coherent coupling Riemann solver. On the other hand, in Subsection 4.1 we proved that the coupling Riemann solver corresponding to the choice $A=D_{v}$ is coherent. In the current subsection we construct a subset $A \supsetneq A_{0}$ which is minimal with respect to inclusion and such that the corresponding coupling Riemann solver is coherent.

The construction of such an $A$ is made as follows. By (H.4) we start from the set $A_{0} \neq \emptyset$ defined in (3.20); recall that $A_{0} \neq D_{v}$ by (3.21). Motivated by the proof of Theorem 4.4, see (4.7), we define a new set $\mathrm{A}_{1} \supsetneq \mathrm{A}_{0}$ as follows: for any $\left(u_{\ell}, u_{r}\right) \in \mathrm{A}_{0}$, whose traces $\left(u_{\mathrm{v}}^{-}, u_{\mathrm{v}}^{+}\right)$satisfy $(4.7)_{2}$, we include $\left(u_{\mathrm{v}}^{-}, u_{\mathrm{v}}^{+}\right)$in $A_{1}$. The procedure is then iterated from $A_{1}$ to obtain $A_{2}$, and so on. Notice that there is a slight abuse of notation: the sets $A_{1}$ and $A_{2}$ considered in this subsection do not coincide with those defined in (3.18) and (3.19).

Summing up, we recursively define the sequence of domains $\mathrm{A}_{n} \subseteq \mathrm{D}_{\mathrm{v}}, n \in \mathbb{N}$, and $\mathrm{A}$ as follows:

$$
\begin{aligned}
\mathrm{A}_{0} & \doteq \mathrm{A}_{0}, \\
\mathrm{~A}_{n+1} & \doteq \mathrm{A}_{n} \cup\left\{\left(u_{\mathrm{v}}^{-}, u_{\mathrm{v}}^{+}\right) \in \mathrm{D}_{\mathrm{v}}:\left(u_{\ell}, u_{r}\right) \in \mathrm{A}_{n} \text { and }\left(u_{\mathrm{v}}^{-}, u_{\mathrm{v}}^{+}\right) \text {satisfies }(4.7)_{2}\right\}, \\
\mathrm{A} & \doteq \bigcup_{n \in \mathbb{N}} \mathrm{A}_{n} .
\end{aligned}
$$

Above we used the notation $u_{\mathrm{v}}^{ \pm} \doteq \mathcal{R} \mathcal{S}_{\mathrm{v}}\left[u_{\ell}, u_{r}\right]\left(0^{ \pm}\right)$. Clearly, we have $\mathrm{A}_{0} \subseteq \mathrm{A}_{n} \subseteq \mathrm{A}_{n+1} \subseteq \mathrm{A} \subseteq \mathrm{D}_{\mathrm{v}}$.

Theorem 4.6 (Coherence). Under (4.8), the coupling Riemann solver $\mathcal{R S}_{\mathrm{v}}$ is coherent.

Proof. We have to check $\left(\mathbf{c h}_{\mathbf{v}}\right)$ for any $\left(u_{\ell}, u_{r}\right) \in \mathrm{D}_{\mathrm{v}}$.

First, take $\left(u_{\ell}, u_{r}\right) \in \mathrm{D}_{\mathrm{v}} \backslash \mathrm{A}$. We claim that also

$$
\left(u_{\mathrm{v}}^{-}, u_{\mathrm{v}}^{+}\right) \in \mathrm{D}_{\mathrm{v}} \backslash \mathrm{A} .
$$


Indeed, by (3.13) we have $u_{\mathrm{v}} \equiv u_{\mathrm{p}}$. As a consequence, $u_{\mathrm{v}}^{ \pm}=u_{\mathrm{p}}^{ \pm}$and by the coherence of $\mathcal{R} \mathcal{S}_{\mathrm{p}}$ we have

$$
\mathcal{R S}_{\mathrm{p}}\left[u_{\mathrm{v}}^{-}, u_{\mathrm{v}}^{+}\right](\xi)=\mathcal{R S}_{\mathrm{p}}\left[u_{\mathrm{p}}^{-}, u_{\mathrm{p}}^{+}\right](\xi)=\left\{\begin{array}{ll}
u_{\mathrm{p}}^{-} & \text {if } \xi<0, \\
u_{\mathrm{p}}^{+} & \text {if } \xi \geq 0,
\end{array}= \begin{cases}u_{\mathrm{v}}^{-} & \text {if } \xi<0 \\
u_{\mathrm{v}}^{+} & \text {if } \xi \geq 0\end{cases}\right.
$$

This implies that $\left(u_{\mathrm{v}}^{-}, u_{\mathrm{v}}^{+}\right)$does not satisfy $(4.7)_{2}$ and therefore $\left(u_{\mathrm{v}}^{-}, u_{\mathrm{v}}^{+}\right) \in\left(\mathrm{D}_{\mathrm{v}} \backslash \mathrm{A}\right) \cup \mathrm{A}_{0}$. It remains to show that $\left(u_{\mathrm{v}}^{-}, u_{\mathrm{v}}^{+}\right) \notin \mathrm{A}_{0}$. By Proposition 3.5 this is equivalent to show that $\mathcal{R} \mathcal{S}_{\mathrm{p}}\left[u_{\mathrm{v}}^{-}, u_{\mathrm{v}}^{+}\right]$satisfies $(3.1)$, namely that the restriction to $\xi<0$ of $\mathcal{R} \mathcal{S}_{\mathrm{p}}\left[u_{\mathrm{v}}^{-}, u_{\mathrm{v}}^{+}\right]$takes values in $\Omega_{-}$and that $\mathcal{R} \mathcal{S}_{\mathrm{p}}\left[u_{\mathrm{v}}^{-}, u_{\mathrm{v}}^{+}\right]\left(0^{-}\right) \in \Omega_{-}$. By (4.10) this is equivalent to show that $u_{\mathrm{v}}^{-} \in \Omega_{-}$. Since $\left(u_{\ell}, u_{r}\right) \in \mathrm{D}_{\mathrm{v}} \backslash \mathrm{A}_{0}$, again by Proposition 3.5 we have $u_{\mathrm{v}}^{-}=\mathcal{R S}_{\mathrm{p}}\left[u_{\ell}, u_{r}\right]\left(0^{-}\right) \in \Omega_{-}$. Therefore, claim (4.9) is proved. By (4.9) and (4.10) we deduce that $\left(\mathbf{c h}_{\mathbf{v}}\right)$ is satisfied because

$$
\mathcal{R S}_{\mathrm{v}}\left[u_{\mathrm{v}}^{-}, u_{\mathrm{v}}^{+}\right](\xi)=\mathcal{R} \mathcal{S}_{\mathrm{p}}\left[u_{\mathrm{v}}^{-}, u_{\mathrm{v}}^{+}\right](\xi)= \begin{cases}u_{\mathrm{v}}^{-} & \text {if } \xi<0, \\ u_{\mathrm{v}}^{+} & \text {if } \xi \geq 0 .\end{cases}
$$

Second, take $\left(u_{\ell}, u_{r}\right) \in \mathrm{A}$. Then there exists $n \in \mathbb{N}$ such that $\left(u_{\ell}, u_{r}\right) \in \mathrm{A}_{n}$. If $\left(u_{\mathrm{v}}^{-}, u_{\mathrm{v}}^{+}\right) \notin \mathrm{A}$, then $\left(u_{\mathrm{v}}^{-}, u_{\mathrm{v}}^{+}\right) \notin \mathrm{A}_{n+1}$ and does not satisfy $(4.7)_{2}$ because $\left(u_{\ell}, u_{r}\right) \in \mathrm{A}_{n}$. Therefore (4.11) holds true again and $\left(\mathbf{c h}_{\mathbf{v}}\right)$ is satisfied. On the other hand, if $\left(u_{\mathrm{v}}^{-}, u_{\mathrm{v}}^{+}\right) \in \mathrm{A}$, then it is sufficient to proceed as in the first part of the proof of Proposition 4.2. This concludes the proof.

Proposition 4.7. The set $\mathrm{A}$ is the smallest subset of $\mathrm{D}_{\mathrm{v}}$ that makes coherent the corresponding coupling Riemann solver $\mathcal{R S}_{\mathrm{v}}$.

Proof. This follows directly from the hypothesis (H.4) (motivated by Proposition 3.5) and the construction (4.8) of A.

In the next proposition we show that the present coupling Riemann solver differs from those introduced in the previous subsections. For notational convenience, we denote by $\mathcal{R S}_{\mathrm{v}}^{1}$ the coupling Riemann solver introduced in Subsection 4.1, and so on.

Proposition 4.8. The coupling Riemann solver $\mathcal{R} \mathcal{S}_{\mathrm{v}}^{3}$ differs from both $\mathcal{R} \mathcal{S}_{\mathrm{v}}^{1}$ and $\mathcal{R} \mathcal{S}_{\mathrm{v}}^{2}$, that is, $\mathrm{A}_{0} \neq \mathrm{A} \neq \mathrm{D}_{\mathrm{v}}$.

Proof. By Theorem 4.4 and Theorem 4.6 we have $\mathcal{R} \mathcal{S}_{\mathrm{v}}^{2} \not \equiv \mathcal{R} \mathcal{S}_{\mathrm{v}}^{3}$. We now prove that $\mathcal{R} \mathcal{S}_{\mathrm{v}}^{1} \not \equiv \mathcal{R} \mathcal{S}_{\mathrm{v}}^{3}$ by showing that $\mathrm{A} \neq \mathrm{D}_{\mathrm{v}}$. More precisely, we show that $\left(u^{-}, u^{+}\right) \doteq\left(u_{-}^{*}, u_{-}^{*}\right) \in \mathrm{D}_{\mathrm{v}} \backslash \mathrm{A}$. First, $u^{+} \in \Omega_{-}$and therefore $\left(u^{-}, u^{+}\right)$does not belong to $\mathrm{A}_{0}$, see (3.21). Second, $\mathcal{R S}_{\mathrm{p}}\left[u^{-}, u^{+}\right] \equiv u^{ \pm}$and therefore $\left(u^{-}, u^{+}\right)$does not belong to $\mathrm{A}_{n}$ for any $n$, see $(4.7)_{2}$. Therefore $\left(u^{-}, u^{+}\right) \in \mathrm{D}_{\mathrm{v}} \backslash \mathrm{A}$.

\section{Technical proofs}

We gather in this section the proofs missing in the previous sections.

\subsection{Proof of Proposition 3.5}

In this subsection we prove Proposition 3.5, namely that $u_{\mathrm{p}}(t, x) \doteq \mathcal{R} \mathcal{S}_{\mathrm{p}}\left[u_{\ell}, u_{r}\right](x / t)$ fails to satisfy $(3.1)$ if and only if $\left(u_{\ell}, u_{r}\right) \in \mathrm{A}_{0} \doteq \mathrm{A}_{1} \cup \mathrm{A}_{2}$, with $\mathrm{A}_{1}$ and $\mathrm{A}_{2}$ given in (3.18) and (3.19), respectively. We first observe that $\Omega_{-} \subsetneq \Omega_{+}$by (H.3), and therefore $u_{\mathrm{p}}$ takes values in $\Omega_{+}$and satisfies (3.1) if and only if

$$
\mathcal{R S}_{\mathrm{p}}\left[u_{\ell}, u_{r}\right](\xi) \in \Omega_{-} \quad \text { if } \xi<0 \text { and } \mathcal{R S}_{\mathrm{p}}\left[u_{\ell}, u_{r}\right]\left(0^{-}\right) \in \Omega_{-} .
$$

To prove that (5.1) is not satisfied if and only if $\left(u_{\ell}, u_{r}\right) \in \mathrm{A}_{0}$ we need the following lemmas.

Lemma 5.1. Let $\left(u_{\ell}, u_{r}\right) \in \mathrm{D}_{\mathrm{v}}$. One of the following conditions holds

$$
\begin{aligned}
\mathcal{R S}_{\mathrm{p}}\left[u_{\ell}, u_{r}\right](\xi) & \in \Omega_{+} \backslash \Omega_{-} \text {for some } \xi<0, \\
\mathcal{R S}_{\mathrm{p}}\left[u_{\ell}, u_{r}\right]\left(0^{-}\right) & \in \Omega_{+} \backslash \Omega_{-},
\end{aligned}
$$

if and only if we have both

$$
u_{r} \in \Omega_{+} \backslash \Omega_{-}
$$

and exactly one of the following two cases occurs, see Figure 6: 
(1) either $\widetilde{u}\left(u_{\ell}, u_{r}\right) \in \Omega_{+} \backslash \Omega_{-}$and $\mathcal{R} \mathcal{S}_{\mathrm{p}}\left[u_{\ell}, \widetilde{u}\left(u_{\ell}, u_{r}\right)\right]$ is a 1-shock with strictly negative speed;

(2) or $\widetilde{u}\left(u_{\ell}, u_{r}\right) \in \Omega_{-}$and $\mathcal{R} \mathcal{S}_{\mathrm{p}}\left[\widetilde{u}\left(u_{\ell}, u_{r}\right), u_{r}\right]$ is a 2-shock with strictly negative speed.

Proof. We refer to Figure 6 (on the left and on the right) for cases (1) and (2). Assume that (5.2) holds true; the same analysis can be applied also for (5.3).

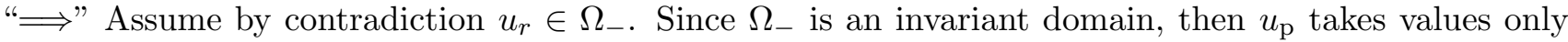
in $\Omega_{-}$, a contradiction. So we have $u_{r} \in \Omega_{+} \backslash \Omega_{-}$.

To prove (1) and (2), we consider the two mutually excluding cases $\widetilde{u}\left(u_{\ell}, u_{r}\right) \in \Omega_{+} \backslash \Omega_{-}$and $\widetilde{u}\left(u_{\ell}, u_{r}\right) \in$ $\Omega_{-}$. Recall that by definition $u_{\mathrm{p}}$ is the juxtaposition of $\mathcal{R S}_{\mathrm{p}}\left[u_{\ell}, \widetilde{u}\left(u_{\ell}, u_{r}\right)\right]$ and $\mathcal{R} \mathcal{S}_{\mathrm{p}}\left[\widetilde{u}\left(u_{\ell}, u_{r}\right), u_{r}\right]$.

(1) Assume $\widetilde{u}\left(u_{\ell}, u_{r}\right) \in \Omega_{+} \backslash \Omega_{-}$. If $\mathcal{R} \mathcal{S}_{\mathrm{p}}\left[u_{\ell}, \widetilde{u}\left(u_{\ell}, u_{r}\right)\right]$ is a 1-rarefaction, then $\widetilde{u}\left(u_{\ell}, u_{r}\right) \in \mathrm{R}_{1}^{u_{\ell}}$ and $\widetilde{\rho}\left(u_{\ell}, u_{r}\right)<$ $\rho_{\ell}$, hence $\widetilde{u}\left(u_{\ell}, u_{r}\right) \in \Omega_{-}$by Proposition 2.3, (L1), a contradiction. If $\mathcal{R} \mathcal{S}_{\mathrm{p}}\left[u_{\ell}, \widetilde{u}\left(u_{\ell}, u_{r}\right)\right]$ is a 1-shock with speed of propagation $\xi_{0} \geqslant 0$, then $\mathcal{R} \mathcal{S}_{\mathrm{p}}\left[u_{\ell}, \widetilde{u}\left(u_{\ell}, u_{r}\right)\right](\xi)=u_{\ell} \in \Omega_{-}$for all $\xi<\xi_{0}$, a contradiction. At last, if $\mathcal{R S}_{\mathrm{p}}\left[u_{\ell}, \widetilde{u}\left(u_{\ell}, u_{r}\right)\right]$ is a 1-shock with speed $\xi_{0}<0$, then $\mathcal{R S}_{\mathrm{p}}\left[u_{\ell}, \widetilde{u}\left(u_{\ell}, u_{r}\right)\right](\xi)=\widetilde{u}\left(u_{\ell}, u_{r}\right) \in \Omega_{+} \backslash \Omega_{-}$ for all $\xi \in\left[\xi_{0}, 0\right)$.

(2) Assume $\widetilde{u}\left(u_{\ell}, u_{r}\right) \in \Omega_{-}$. Then $\mathcal{R S}_{\mathrm{p}}\left[u_{\ell}, \widetilde{u}\left(u_{\ell}, u_{r}\right)\right]$ takes values in $\Omega_{-}$and so there exists $\xi_{*}<0$ such that

$$
\mathcal{R S}_{\mathrm{p}}\left[\widetilde{u}\left(u_{\ell}, u_{r}\right), u_{r}\right]\left(\xi_{*}\right) \in \Omega_{+} \backslash \Omega_{-} .
$$

If $\mathcal{R} \mathcal{S}_{\mathrm{p}}\left[\widetilde{u}\left(u_{\ell}, u_{r}\right), u_{r}\right]$ is a 2-rarefaction then it takes values in

$$
\left\{u \in \mathrm{R}_{2}^{\widetilde{u}\left(u_{\ell}, u_{r}\right)}: \widetilde{\rho}\left(u_{\ell}, u_{r}\right) \leqslant \rho \leqslant \rho_{r}\right\} \subset \Omega_{+} .
$$

We observe that

$$
\left\{u \in \mathrm{R}_{2}^{\widetilde{u}\left(u_{\ell}, u_{r}\right)}: \widetilde{\rho}\left(u_{\ell}, u_{r}\right) \leqslant \rho \leqslant \rho_{r}\right\} \backslash \Omega_{-}=\left\{u \in \mathrm{R}_{2}^{\widetilde{u}\left(u_{\ell}, u_{r}\right)}: \widetilde{\rho}\left(u_{-}^{*}, \widetilde{u}\left(u_{\ell}, u_{r}\right)\right)<\rho \leqslant \rho_{r}\right\}
$$

and

$$
0 \leqslant \widetilde{q}\left(u_{-}^{*}, \widetilde{u}\left(u_{\ell}, u_{r}\right)\right)<q_{r}
$$

imply $\mathcal{R S}_{\mathrm{p}}\left[\widetilde{u}\left(u_{\ell}, u_{r}\right), u_{r}\right](\xi)=\widetilde{u}\left(u_{\ell}, u_{r}\right) \in \Omega_{-}$for any $\xi<0$, a contradiction with (5.4).

If $\mathcal{R S}_{\mathrm{p}}\left[\widetilde{u}\left(u_{\ell}, u_{r}\right), u_{r}\right](\xi)$ is a 2 -shock with speed of propagation $\xi_{0} \geqslant 0$, then $\mathcal{R S}_{\mathrm{p}}\left[\widetilde{u}\left(u_{\ell}, u_{r}\right), u_{r}\right](\xi)=$ $\widetilde{u}\left(u_{\ell}, u_{r}\right) \in \Omega_{-}$for all $\xi<\xi_{0}$, which contradicts (5.4).

At last, if $\mathcal{R S}_{\mathrm{p}}\left[\widetilde{u}\left(u_{\ell}, u_{r}\right), u_{r}\right]$ is a 2 -shock with speed of propagation $\xi_{0}<0$, then $\mathcal{R S}_{\mathrm{p}}\left[\widetilde{u}\left(u_{\ell}, u_{r}\right), u_{r}\right](\xi)=$ $u_{r} \in \Omega_{+} \backslash \Omega_{-}$for all $\xi \in\left[\xi_{0}, 0\right)$.

"£" The converse implication easily follows because we considered above all possible cases.

Lemma 5.2. We have

$$
\left\{\left(u_{\ell}, u_{r}\right) \in \mathrm{D}_{\mathrm{v}}: \text { either (5.2) or (5.3) holds }\right\}=\mathrm{A}_{0} .
$$

Proof. By Lemma 5.1 we have that $\left(u_{\ell}, u_{r}\right) \in \mathrm{D}_{\mathrm{v}}$ is such that either (5.2) or (5.3) holds if and only if $u_{r} \in \Omega_{+} \backslash \Omega_{-}$and exactly one of the cases (1) and (2) described in Lemma 5.1 occurs. It is therefore clear that it is sufficient to prove that the sets $A_{1}$ and $A_{2}$ defined in (3.18), (3.19) coincide with the sets of the pairs $\left(u_{\ell}, u_{r}\right)$ that satisfy respectively (1) and (2). This is is obvious for $\mathrm{A}_{2}$.

About $\mathrm{A}_{1}$, it is sufficient to observe that $\widetilde{u}\left(u_{\ell}, u_{r}\right) \in \Omega_{+} \backslash \Omega_{-}$implies that $\mathcal{R S}_{\mathrm{p}}\left[u_{\ell}, \widetilde{u}\left(u_{\ell}, u_{r}\right)\right]$ is a 1-shock with negative speed. Indeed, we have $\left\{u \in \mathrm{R}_{1}^{u_{\ell}}: \rho \leqslant \rho_{\ell}\right\} \subset \Omega_{-}$, which implies that $\mathcal{R S}_{\mathrm{p}}\left[u_{\ell}, \widetilde{u}\left(u_{\ell}, u_{r}\right)\right]$ has to be a 1-shock. Moreover, the inequalities $\widetilde{q}\left(u_{\ell}, u_{r}\right)<\widetilde{q}\left(u_{\ell}, u_{-}^{*}\right) \leqslant q_{\ell}$ imply that the shock speed is negative.

We now prove Proposition 3.5. Obviously $u_{\mathrm{p}}$ fails to satisfy (3.1) (in $\mathbb{R}_{-}$) if and only if either (5.2) or (5.3) is satisfied. By Lemma 5.1 the requirement of either (5.2) or (5.3) is equivalent to take $u_{r} \in \Omega_{+} \backslash \Omega_{-}$ and that either (1) or (2) are satisfied. At last, by Lemma 5.2 this is equivalent to have $\left(u_{\ell}, u_{r}\right) \in \mathrm{A}_{0}$. This concludes the proof of Proposition 3.5. 


\subsection{Proof of Proposition 3.6}

Here we prove Proposition 3.6, namely that $\mathcal{R S}_{\mathrm{v}}$ given in Definition 3.3 is a coupling Riemann solver in the sense of Definition 3.2. We must show that for any $\left(u_{\ell}, u_{r}\right) \in \mathrm{D}_{\mathrm{v}}$ the function $u_{\mathrm{v}}$ is a coupling solution to Riemann problem (2.1), (2.2) in the sense of Definition 3.1. To this aim it is sufficient to show that

$$
\begin{gathered}
q_{\mathrm{v}}\left(t, 0^{-}\right)=q_{\mathrm{v}}\left(t, 0^{+}\right) \quad \text { for a.e. } t>0, \\
u_{\mathrm{v}}(t, \pm x) \in \Omega_{ \pm} \quad \text { and } \quad u_{\mathrm{v}}\left(t, 0^{ \pm}\right) \in \Omega_{ \pm} \quad \text { if } x>0 \text { and } t>0,
\end{gathered}
$$

the remaining conditions being evident.

We first prove condition (5.5). If $\mathcal{R S}_{\mathrm{v}}$ is given by (3.12), then (5.5) follows by (3.17). If $\mathcal{R} \mathcal{S}_{\mathrm{v}}$ is given by (3.13), then it is sufficient to observe that $u_{\mathrm{v}} \equiv u_{\mathrm{p}}$ and that $u_{\mathrm{p}}$ satisfies (5.5) by the first Rankine-Hugoniot condition (2.3).

For the proof of (5.6) we first observe that $\Omega_{-} \subsetneq \Omega_{+}$by $\left(\right.$H.3). As a consequence $u_{\mathrm{v}}$ takes values in $\Omega_{+}$ for any $\left(u_{\ell}, u_{r}\right) \in \mathrm{D}_{\mathrm{v}}$. Therefore, $u_{\mathrm{v}}$ satisfies (5.6) if and only if

$$
\mathcal{R S}_{\mathrm{v}}\left[u_{\ell}, u_{r}\right](\xi) \in \Omega_{-} \quad \text { if } \xi<0 \quad \text { and } \quad \mathcal{R S}_{\mathrm{V}}\left[u_{\ell}, u_{r}\right]\left(0^{-}\right) \in \Omega_{-} .
$$

We have two cases.

(i) If $\left(u_{\ell}, u_{r}\right) \in \mathrm{D}_{\mathrm{v}} \backslash \mathrm{A}$, then $u_{\mathrm{v}} \equiv u_{\mathrm{p}}$ and $\left(u_{\ell}, u_{r}\right) \in \mathrm{D}_{\mathrm{v}} \backslash \mathrm{A}_{0}$; hence (5.7) reduces to (5.1), which holds by what we proved in Subsection 5.1.

(ii) If $\left(u_{\ell}, u_{r}\right) \in \mathrm{A}$, by (H.2) and (3.10) we deduce

$$
Q_{\mathrm{A}}\left(u_{\ell}, u_{r}\right) \in I_{\mathrm{A}}\left(u_{\ell}, u_{r}\right)=\left[\underline{Q}\left(u_{r}\right), \bar{Q}\left(u_{\ell}\right)\right] \cap\left[Q_{1}\left(u_{\ell}\right), Q_{2}\left(u_{r}\right)\right] .
$$

Denote $\hat{u}_{\mathrm{A}}=\hat{u}_{\mathrm{A}}\left(u_{\ell}, u_{r}\right)$ and $\check{u}_{\mathrm{A}}=\check{u}_{\mathrm{A}}\left(u_{\ell}, u_{r}\right)$. Condition (3.5) ensures that $\mathcal{R S}_{\mathrm{p}}\left[u_{\ell}, \hat{u}_{\mathrm{A}}\right]$ takes values in $\mathrm{FL}_{1}^{u_{\ell}}$ and $\mathcal{R S}_{\mathrm{p}}\left[\check{u}_{\mathrm{A}}, u_{r}\right]$ in $\mathrm{FL}_{2}^{\check{u}_{\mathrm{A}}}$; the former is a single wave contained in $x \leq 0$ and the latter is a single wave contained in $x \geq 0$. Condition (3.7) implies $\hat{u}_{\mathrm{A}} \in \Omega_{-}$and $\check{u}_{\mathrm{A}} \in \Omega_{+}$. Then (5.7) holds true.

This concludes the proof of Proposition 3.6.

\subsection{Remark on the proof of Proposition 4.2}

We show that a possible case in the proof of Proposition 4.2 does not occur, indeed. We postponed this result in this subsection because that proof does not depend on this clarification. However, this remark is interesting because it shows a kind of "stability" for $\mathcal{R} \mathcal{S}_{\mathrm{v}}$ which follows from the choice (4.1) for $Q_{\mathrm{A}}$.

Proposition 5.3. If both $\left(u_{\ell}, u_{r}\right)$ and $\left(u_{\mathrm{v}}^{-}, u_{\mathrm{v}}^{+}\right)$belong to $\mathrm{A}$, then

$$
\hat{u}_{\mathrm{A}}\left(u_{\ell}, u_{r}\right)=\hat{u}_{\mathrm{A}}\left(u_{\mathrm{v}}^{-}, u_{\mathrm{v}}^{+}\right) .
$$

Proof. Assume by contradiction that $\hat{u}_{\mathrm{A}}\left(u_{\ell}, u_{r}\right) \neq \hat{u}_{\mathrm{A}}\left(u_{\mathrm{v}}^{-}, u_{\mathrm{v}}^{+}\right)$. By Lemma 4.1 we have $Q_{\mathrm{A}}\left(u_{\mathrm{v}}^{-}, u_{\mathrm{v}}^{+}\right)=q_{\mathrm{v}}^{ \pm}=$ $Q_{\mathrm{A}}\left(u_{\ell}, u_{r}\right)$. As a consequence, see Figure 10, we have

$$
0<\hat{q}_{\mathrm{A}}\left(u_{\ell}, u_{r}\right)=\mathcal{S}_{1}^{u_{\ell}}\left(\hat{\rho}_{\mathrm{A}}\left(u_{\ell}, u_{r}\right)\right)<q_{\ell}, \quad 0<\rho_{\ell}<\hat{\rho}_{\mathrm{A}}\left(u_{\ell}, u_{r}\right)<\hat{\rho}_{\mathrm{A}}\left(u_{\mathrm{v}}^{-}, u_{\mathrm{v}}^{+}\right), \quad a<\hat{v}_{\mathrm{A}}\left(u_{\ell}, u_{r}\right)<v_{\ell}
$$

By $(2.5)_{1}$ and $(5.8)_{1}$ we have

$$
\begin{aligned}
& \hat{q}_{\mathrm{A}}\left(u_{\ell}, u_{r}\right)=\hat{\rho}_{\mathrm{A}}\left(u_{\ell}, u_{r}\right)\left[v_{\ell}-a\left(\sqrt{\frac{\hat{\rho}_{\mathrm{A}}\left(u_{\ell}, u_{r}\right)}{\rho_{\ell}}}-\sqrt{\frac{\rho_{\ell}}{\hat{\rho}_{\mathrm{A}}\left(u_{\ell}, u_{r}\right)}}\right)\right]<q_{\ell}=\rho_{\ell} v_{\ell} \\
\Longleftrightarrow & \left(\hat{\rho}_{\mathrm{A}}\left(u_{\ell}, u_{r}\right)-\rho_{\ell}\right) v_{\ell}<\left(\hat{\rho}_{\mathrm{A}}\left(u_{\ell}, u_{r}\right)-\rho_{\ell}\right) a \sqrt{\frac{\hat{\rho}_{\mathrm{A}}\left(u_{\ell}, u_{r}\right)}{\rho_{\ell}}},
\end{aligned}
$$




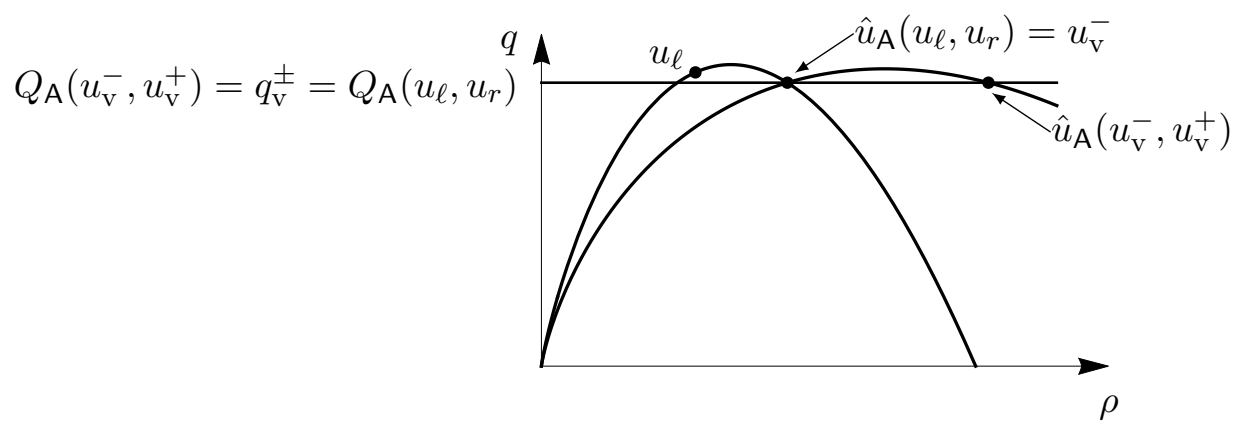

Figure 10: The above Lax curves are not exact and are meant just to help in the exposition of the proof by contradiction of Proposition 5.3.

and then, by $(5.8)_{2}$,

$$
v_{\ell}<a \sqrt{\frac{\hat{\rho}_{\mathrm{A}}\left(u_{\ell}, u_{r}\right)}{\rho_{\ell}}}
$$

This together with $(5.8)_{3}$ implies

$$
a<\hat{v}_{\mathrm{A}}\left(u_{\ell}, u_{r}\right)=v_{\ell}-a\left(\sqrt{\frac{\hat{\rho}_{\mathrm{A}}\left(u_{\ell}, u_{r}\right)}{\rho_{\ell}}}-\sqrt{\frac{\rho_{\ell}}{\hat{\rho}_{\mathrm{A}}\left(u_{\ell}, u_{r}\right)}}\right)<a \sqrt{\frac{\rho_{\ell}}{\hat{\rho}_{\mathrm{A}}\left(u_{\ell}, u_{r}\right)}} \Longrightarrow \hat{\rho}_{\mathrm{A}}\left(u_{\ell}, u_{r}\right)<\rho_{\ell},
$$

which contradicts $(5.8)_{2}$.

\section{Conclusions}

Along the lines of $[7,8]$, in this paper we introduced a model for the gas flow through a valve. The noteworthy aspects of the modeling are two: first, the search for invariant domains of the solutions, which guarantee the flow to take place within prescribed bounds of pressure and flux; second, the presence of a valve that maximizes the flux. The main issue was the characterization of the coherence of the solvers, which is the basis for the study of chattering. We examined three types of valves: for two of them coherence was granted while it was disproved for the other one.

The mechanisms that lead to the loss of coherence, and then possibly trigger chattering, are not yet fully clear, despite the efforts in this paper and in $[7,8]$, and this demands for further investigations. Indeed, it seems that such a behavior depends so strongly on the type of valve under consideration that it could be difficult, if not impossible, to establish general criteria.

For instance, in the case of the pressure regulators considered in [7], chattering cannot arise if the valve is initially active [7, Theorem 4.1]; this happens in particular if the valve is closed. On the other hand, in the case of the pressure independent control valves studied in [8], chattering may arise even if the valve is initially active; more precisely, it may arise only if the valve is initially closed [8, Corollary 4.6]. The latter conclusion is valid in an explicit example of a retarded valve given in $[8, \S 6]$. Also the example of incoherence constructed in Theorem 4.4 of the current paper concerns a valve that is initially active.

The next steps in the modeling are now about the existence of global (in time) solutions for $\mathbf{B V}$ initial data, and the analysis in these solutions about when and how chattering arises. This program will be accomplished in forthcoming papers, where also numerical simulations will be provided. A side problem is the study of coherence for the case when a compressor replaces the valve [15]; in this case, the coherence of the corresponding coupling Riemann solver has not yet been investigated.

\section{Acknowledgments}

The authors are members of GNAMPA. They were partially supported by the INdAM-GNAMPA Project 2019 "Equazioni alle derivate parziali di tipo iperbolico o non locale ed applicazioni". 


\section{References}

[1] D. Amadori. Initial-boundary value problems for nonlinear systems of conservation laws. NoDEA Nonlinear Differential Equations Appl., 4(1):1-42, 1997.

[2] M. K. Banda, M. Herty, and A. Klar. Coupling conditions for gas networks governed by the isothermal Euler equations. Netw. Heterog. Media, 1(2):295-314, 2006.

[3] M. K. Banda, M. Herty, and A. Klar. Gas flow in pipeline networks. Netw. Heterog. Media, 1(1):41-56, 2006.

[4] C. Bardos, A. Y. le Roux, and J.-C. Nédélec. First order quasilinear equations with boundary conditions. Comm. Partial Differential Equations, 4(9):1017-1034, 1979.

[5] A. Bressan. Hyperbolic systems of conservation laws, volume 20. Oxford University Press, Oxford, 2000.

[6] R. M. Colombo and M. Garavello. Comparison among different notions of solution for the $p$-system at a junction. Discrete Contin. Dyn. Syst., Supplement:181-190, 2009.

[7] A. Corli, M. Figiel, A. Futa, and M. D. Rosini. Coupling conditions for isothermal gas flow and applications to valves. Nonlinear Anal. Real World Appl., 40:403-427, 2018.

[8] A. Corli and M. D. Rosini. Coherence and chattering of a one-way valve. Accepted on Zeit. Ang. Math. Mech., 2019.

[9] C. M. Dafermos. Hyperbolic conservation laws in continuum physics. Springer-Verlag, Berlin, fourth edition, 2016.

[10] E. Dal Santo, C. Donadello, S. F. Pellegrino, and M. D. Rosini. Representation of capacity drop at a road merge via point constraints in a first order traffic model. ESAIM Math. Model. Numer. Anal., 53(1):1-34, 2019.

[11] E. Dal Santo, M. D. Rosini, N. Dymski, and M. Benyahia. General phase transition models for vehicular traffic with point constraints on the flow. Math. Methods Appl. Sci., 40(18):6623-6641, 2017.

[12] N. S. Dymski, P. Goatin, and M. D. Rosini. Existence of BV solutions for a non-conservative constrained Aw-Rascle-Zhang model for vehicular traffic. to appear on Journal of Mathematical Analysis and Applications, 2018.

[13] M. Garavello and B. Piccoli. Traffic flow on a road network using the Aw-Rascle model. Comm. Partial Differential Equations, 31(1-3):243-275, 2006.

[14] M. Gugat, G. Leugering, A. Martin, M. Schmidt, M. Sirvent, and D. Wintergerst. MIP-based instantaneous control of mixed-integer PDE-constrained gas transport problems. Comput. Optim. Appl., 70(1):267-294, 2018.

[15] M. Herty. Modeling, simulation and optimization of gas networks with compressors. Netw. Heterog. Media, $2(1): 81-97,2007$.

[16] C. Hős and A. R. Champneys. Grazing bifurcations and chatter in a pressure relief valve model. Phys. D, 241(22):2068-2076, 2012.

[17] D. Hoff. Invariant regions for systems of conservation laws. Trans. Amer. Math. Soc., 289(2):591-610, 1985.

[18] T. Koch, B. Hiller, M. E. Pfetsch, and L. Schewe, editors. Evaluating gas network capacities, volume 21 of MOS-SIAM Series on Optimization. Society for Industrial and Applied Mathematics (SIAM), Philadelphia, PA, 2015.

[19] P. G. LeFloch. Hyperbolic systems of conservation laws. Lectures in Mathematics ETH Zürich. Birkhäuser Verlag, Basel, 2002.

[20] R. J. LeVeque. Numerical methods for conservation laws. Birkhäuser Verlag, Basel, 1990.

[21] G. Licskó, A. Champneys, and C. Hös. Nonlinear analysis of a single stage pressure relief valve. IAENG Int. J. Appl. Math., 39(4):286-299, 2009.

[22] A. Martin, M. Möller, and S. Moritz. Mixed integer models for the stationary case of gas network optimization. Math. Program., 105(2-3, Ser. B):563-582, 2006.

[23] M. Möller. Mixed Integer Models for the Optimisation of Gas Networks in the Stationary Case. PhD thesis, Fach. Mathematik T.U. Darmstadt, 2004. 